\title{
NUCLEUS-NUCLEUS COLLISIONS AT ULTRA-RELATIVISTIC ENERGIES: STATUS AND PROSPECTS
}

\author{
F. Plasil
}

Oak Ridge National Laboratory, Oak Ridge, Tennessee 37831-6372, USA

\section{- Invited Lecture -}

\section{Relativistic Heavy-Ion Physics}

International School/Workshop for Young Physicists

Prague, Czech Republic, September 19-23, 1994

\footnotetext{
"The submitted manuscript has been authored by a contractor of the U.S. Government under contract DE-AC05-84OR21400. Accordingly, the U.S. Government retains a nonexclusive, royalty-free license to publish or reproduce the published form of this contribution, or allow others to do so, for U.S. Government purposes."
}

\begin{abstract}
DISCLAIMER
This report was prepared as an account of work sponsored by an agency of the United States Government. Neither the United States Government nor any agency thereof, nor any of their employees, makes any warranty, express or implied, or assumes any legal liability or responsibility for the accuracy, completeness, or usefulness of any information, apparatus, product, or process disclosed, or represents that its use would not infringe privately owned rights. Reference herein to any specific commercial product, process, or service by trade name, trademark, manufacturer, or otherwise does not necessarily constitute or imply its endorsement, recommendation, or favoring by the United States Government or any agency thereof. The views and opinions of authors expressed herein do not necessarily state or reflect those of the United States Government or any agency thereof.
\end{abstract}




\section{DISCLAIMER}

Portions of this document may be illegible in electronic image products. Images are produced from the best available original document. 


\title{
NUCLEUS-NUCLEUS COLLISIONS AT ULTRA-RELATIVISTIC ENERGIES: STATUS AND PROSPECTS
}

\author{
F. PLASIL \\ Oak Ridge National Laboratory, ${ }^{1}$ Oak Ridge, Tennessee 37831-6372, USA
}

\section{Introduction}

This paper is based on three lectures presented at the Prague Seminar on Relativistic Heavy-Ion Physics in September 1994. The first lecture, following a general introduction, focuses on three different aspects of the CERN experiment WA80. I first present results on global event characteristics deduced primarily from measured distributions of transverse energy and of forward energy [1]. The purpose is to introduce the main general features of nucleus-nucleus reactions at the highest energies currently available. I highlight the role of projectile-target geometry, discuss the degree of nuclear stopping, and estimate the energy densities attained in these reactions. This discussion is followed by a presentation of one of two topics that are unique to the WA80 experiment and which are not addressed by any of the other CERN collaborations that study nucleus-nucleus reactions: direct measurements of photons [2]. The second topic unique to WA80, measurements of proton-proton correlations in the target-fragmentation region [3], is covered in the first part of the second lecture. The remainder of the second lecture is devoted to a selective overview of results obtained at the AGS accelerator of Brookhaven National Laboratory (BNL). The third lecture is devoted to a discussion of the two main experiments, STAR and PHENIX, planned for the Relativistic Heavy Ion Collider, RHIC, under construction at BNL.

\section{Global event characteristics at CERN SPS energies}

Global event properties are studied by determining, on an event-by-event basis, the number of particles emitted and/or the energy they carry. No attempt is made to identify particles, and the results are presented as a function of pseudorapidity, $\eta$. The WA80 data were obtained from a number of CERN/SPS runs between 1986 and 1990. The initial beams available were ${ }^{16} \mathrm{O}$ nuclei at 60 and $200 \mathrm{GeV} /$ nucleon. Later runs made use of ${ }^{32} \mathrm{~S}$ ions at $200 \mathrm{GeV} /$ nucleon. The availability of two different projectile nuclei and two different bombarding energies, together with the use of a number of different targets, enables us to study the gross features of the reactions in a systematic way. Our results are obtained from measurements of the energy detected in the forward direction, $E_{F}$, and the transverse energy, $E_{T}$, which is defined as the sum of the transverse masses of all interacting and produced particles. Both energies are, in some sense, a measure of the degree to which an interaction has (or has not) taken place, and as may be expected,

\footnotetext{
${ }^{1}$ Managed by Martin Marietta Energy Systems, Inc., under contract DE-AC05-84OR21400 with the U.S. Department of Energy
} 
they are strongly anti-correlated. Both $E_{F}$ and $E_{T}$ were measured by means of sampling calorimeters $[4,5]$.

The main reaction features can be understood on the basis of a schematic "cleancut" geometry picture in which colliding nuclei are regarded, to first order, as colliding spheres. At any given impact parameter, all nucleons that are contained in the overlap region of the two spheres are considered to be participants, while nucleons outside of this region are spectators. On the assumption that $E_{F}$ depends monotonically on the impact parameter $b$, it is possible to deduce $b$ on an event-by-event basis. Furthermore, based on Glauber theory $[1,6]$, it is also possible to deduce, for each event, the number of projectile nucleons participating in the collision, $W_{p}$, as well as the number of target participants, $W_{T}$. As will be seen below, the determination of the total number of participants provides us with a valuable tool which enables us to obtain a unified quantitative description of the various reactions studied by WA80.

Transverse energy measurements were performed with the Midrapidity Calorimeter MIRAC [5]. $E_{T}$ measurements are important because results can be used to estimate energy densities that might have been attained (see below) and because they provide us with an indication of the degree of nuclear stopping that has taken place. Most $E_{T}$ distributions, including those of WA80, are measured in a limited pseudorapidity region. Comparisons between different experiments (and between results from the same experiment at different energies) can be made in terms of $\frac{d E_{T}}{d \eta}$ distributions. Such distributions from ${ }^{32} \mathrm{~S}$ interactions with various target nuclei are shown in Fig. 1. It can be seen (and it is confirmed by numerous other results) that these distributions are Gaussian. Consequently, we can study the systematics of $E_{T}$ production with the help of three parameters which characterize each measured distribution: the maximum value, the average, and the width.

As an example, we show in Fig. 2 the maximum values of $\frac{d E_{r}}{d \eta}$ distributions as a function of the average total number of participants, $\bar{W}$. The $\left.\frac{d E_{T}}{d \eta}\right|_{\max }$ values are seen to be nearly proportional to $\bar{W}$ with slopes of 0.64 and $0.9 \mathrm{GeV} /$ nucleon at $60 \mathrm{~A} \mathrm{GeV}$ and $200 \mathrm{~A} \mathrm{GeV}$, respectively. The increase of $\left.\frac{d E_{r}}{d \eta}\right|_{\max }$ as a function of increasing target mass noted in Fig. 1 is thus a result of the increasing number of target participants involved in the collision. Similar increases in $\left.\frac{d E_{T}}{d \eta}\right|_{\max }$ are observed as a function of the centrality of the collision and as a function of projectile mass, both of which also involve an increase in the total number of participants. The interesting overall conclusion is that the transverse energy produced per participating baryon depends only on the beam energy per nucleon and not on projectile mass or on the centrality of the collision.

We next consider the degree of nuclear stopping involved in the collisions. This is an important concept since a high degree of stopping is a prerequisite for the formation of a baryon-rich system of sufficient energy density such that it may lead to a phase transition to the quark-gluon plasma (QGP) in the currently-explored fixed-target systems. While an intuitive picture of this concept is not difficult to grasp, no universally accepted quantitative definition of it exists. Stopping has been alternatively described as the "boosting of participant nucleons to central rapidity," as the "rapidity loss of projectile nucleons," and, in an effort to obtain a quantitative description based on readily-available 
data, as the "transverse energy produced relative to that which can be expected on the basis of some (e.g., fireball) model in which full stopping is implicit."

Since in WA80 experiments the participating baryons are not specifically identified, we are restricted to the last definition given above in estimating the degree of stopping. We first obtain values for total $E_{T}$ from the $\frac{d E_{T}}{d \eta}$ data by integrating the Gaussian distributions which have been extrapolated into the region of $\eta$ in which measurements were not made. We then proceed to obtain values of the stopping ratio, $R_{T}$, by dividing the measured $E_{T}$ values by $\frac{\pi}{4}\left(E_{c . m .}-\bar{W} \mathrm{mc}^{2}\right)$, which is the upper limit on possible production of transverse energy, based on the formation of an isotropic fireball. This leads to a lower limit on the estimated degree of nuclear stopping. The $R_{T}$ values obtained by this procedure range from $\sim 0.6$ for reactions at $60 \mathrm{GeV} /$ nucleon to $\sim 0.5$ for reactions at $200 \mathrm{GeV}$. The assumption of a Landau fireball, which includes longitudinal expansion not present in an isotropic fireball, leads to somewhat higher values of the stopping ratio. However, the overall conclusion remains that the degree of stopping at these energies is high, but not complete. These are reasonably favorable conditions for high energy-density production, which is the next topic that we consider.

In the case of energy densities there is no problem of definition (energy per unit volume, expressed in $\mathrm{GeV} / \mathrm{fm}^{3}$ ). Here, however, there is a conceptual problem when attempts are made to extract energy density values from data. To what stage of the collision process do the extracted values refer? During the course of a reaction, there is first a buildup of energy density during the early stages, followed by a decrease during the expansion process. The buildup of energy density results from both the dynamics of the collision (sharp increase in baryon density per unit volume) as well as from particle formation (mostly pions). Yet, only educated guesses are available for the "formation time" (e.g., $1 \mathrm{fm} / \mathrm{c}$ ), and it remains virtually a free parameter. It is even possible that the expansion process is initiated before particle formation is terminated. The most frequently-used method of estimating the attained energy density is attributed to Bjorken [7]. It assumes longitudinal growth and free hydrodynamic flow in a baryon-free region. Strictly speaking, given the assumptions of the Bjorken approach, it should only be applied to cases where there is a baryon-free plateau in the $\frac{d E_{T}}{d \eta}$ distribution and not to the Gaussian distributions with large midrapidity baryon admixtures obtained here. This reservation notwithstanding, values of $\epsilon_{B J}$ for our cases range from a low of $1.0 \mathrm{GeV} / \mathrm{fm}^{3}$ for ${ }^{16} \mathrm{O}+\mathrm{Cu}$ at $60 \mathrm{GeV} /$ nucleon to $2.0 \mathrm{GeV} / \mathrm{fm}^{3}$ for ${ }^{32} \mathrm{~S}+\mathrm{Au}$ at 200 $\mathrm{GeV} /$ nucleon. In the latter case, if a sample of events with the highest values of $E_{T}$ is selected, a value of $2.9 \mathrm{GeV} / \mathrm{fm}^{3}$ is obtained for $\epsilon_{B J}$. Theoretical estimates of the energy density required for the phase transition to the QGP to take place are typically in the range of $3 \mathrm{GeV} / \mathrm{fm}^{3}$ with a broad band of uncertainty around this value. Thus if the required energy densities for QGP formation have not actually been attained in our cases, we probably do not stand very far from this goal. 


\section{WA80 photon measurements}

Photons, being color-neutral, are potentially good probes of highly compressed and excited nuclear matter and of possible transitions to the quark-gluon plasma. Unfortunately, measurements of photons from nucleus-nucleus collisions are difficult due to a huge combinatorial background from the decay of $\pi^{0}$ 's (and of other mesons) and also due to the background resulting from hadronic showers produced by a large number of charged pions impinging on the photon detectors [8]. However, through the application of the event-mixing technique in which combinatorial backgrounds are evaluated by deliberately pairing detected photons from separate events, it is possible to reconstruct $\pi^{0}$ and $\eta$ mesons down to signal-to-background ratios of 0.002 . Absolute $\pi^{0}$ transverse momentum spectra are shown in Fig. 3 for central and peripheral events from $S+S$ and $\mathrm{S}+\mathrm{Au}$ reactions at $200 \mathrm{GeV} /$ nucleon. The data cover a range of seven orders of magnitude. Exponential fits to the spectra in the $p_{T}$ range from about 0.5 to $2.0 \mathrm{GeV} / \mathrm{c}$ are also shown in Fig. 3, together with the corresponding slope parameters. In Fig. $4 \eta / \pi^{0}$ ratios are shown as a function of $p_{T}$, together with results from similar hadron-hadron and hadron-nucleus measurements [9]. The solid line is the result of a fit to all data shown in Fig. 4 following the $m_{T}$-scaling parametrization of Bourquin and Gaillard [10]. We conclude that $m_{T}$ scaling of meson production in nucleus-nucleus collisions is consistent with the phenomenological $m_{T}$ scaling previously established in hadron-hadron and hadron-nucleus reactions. We make use of this conclusion to estimate the small contribution that decays of hadrons, other than the measured $\pi^{0}$ 's and $\eta$ mesons, make to the total observed yield of photons.

We next consider yields of single photons. Single "direct" photons are expected from a variety of different sources such as hard QCD processes and thermal radiation from the QGP. Specific processes include quark-quark annihilation and quark-gluon Compton scattering. In a low-temperature hadron gas the photons are produced primarily via the $\pi^{+}+\pi^{-} \rightarrow \gamma+\rho^{0}$ and the $\pi^{ \pm}+\rho \rightarrow \gamma+\pi^{ \pm}$reactions. For a given event class, the single photon yield is obtained from the difference between the measured inclusive photon yield for that event class and the photon yield expected from the decay of known resonances for the same event class obtained with the aid of simulations. The input to the simulation calculations is obtained from our measured $\pi^{0}$ and $\eta$ cross sections and from estimates (based on $m_{T}$ scaling) of the contributions to the total gamma yield from the decay of nonreconstructed mesons, such as $\omega, \eta^{\prime}$, and $K^{0}$. The total contribution from the decay of nonreconstructed mesons is estimated to be less than $2 \%$ of the contribution from reconstructed hadrons. The range of total errors is $6-7 \%$ in the $p_{T}$ range $0.3-1.5$ $\mathrm{GeV} / \mathrm{c}$.

Traditionally, single-photon results are presented in terms of $\gamma / \pi^{0}$ ratios since most observed photons arise from $\pi^{0}$ decay, and therefore, the $\pi^{0}$ yield sets the scale for the sensitivity of the direct photon search. Plots of $\gamma / \pi^{0}$ versus $p_{T}$ indicate that a small, but statistically-significant excess of photons over those due to hadronic decays is observed in central collisions but not in peripheral collisions. In Fig. 5 the preliminary invariant cross section of single photons from central $\mathrm{S}+\mathrm{Au}$ collisions is shown as a function of $p_{T}$. 
It can be seen that the slope parameter is $180 \mathrm{MeV} / \mathrm{c}$. Based on the decay kinematics of assumed single-photon production, this translates to a hadronic gas temperature of about $165 \mathrm{MeV}$. This result, even though preliminary, has aroused considerable interest in the theoretical community $[11,12]$. Work continues via independent evaluations in order to confirm or modify the preliminary WA80 single-photon yields presented here.

\section{Two-proton correlations in the target fragmentation region}

In this first part of the second lecture, we consider the most interesting results that have been obtained by WA80 in the target fragmentation region $(-1.7<\eta<1.3)$. These are the source radii extracted from measured two-proton correlations [3]. The principle involved here is based on the interference that arises between matter waves of two identical particles if there are two possible paths for the particles to follow from the emission point to the coincident detectors. The observed amplitudes lead to a separation of the emission regions, and source distributions can then be deduced. In our case, no reliable analytical expressions exist with which direct fits to the data can be made. Consequently, following Pratt [13], our results are deduced from comparisons of numerical simulations to data. We have assumed spherical Gaussian-shaped source distributions with a lifetimes of $\tau=0 \mathrm{fm} / \mathrm{c}$. This results in upper limits for spatial extents of extracted sources. Detector effects are included in the calculations, and care was taken to correct for misidentified particles and for other experimental effects.

Extracted radii obtained by this procedure are shown in Fig. 6 for reactions of protons and ${ }^{16} \mathrm{O}$ ions with $\mathrm{C}, \mathrm{Cu}, \mathrm{Ag}$, and $\mathrm{Au}$ targets, as well as for ${ }^{32} \mathrm{~S}$ interactions with $\mathrm{Al}$ and $\mathrm{Au}$ nuclei at $200 \mathrm{GeV} /$ nucleon. Remarkably, the radii follow closely a monotonic increase with the mass of the target, proportional to $A_{\text {Target }}^{1 / 3}$. The hatched band in Fig. 6 indicates geometric radii of the target nuclei. The source radii extracted from heavy-ion reactions lie somewhat above the target geometric radii, while those obtained from $p$-induced reactions are very close to the target radii. This suggests that, at these energies, even in proton-induced reactions, the whole target nucleus is involved in the reaction. We conclude that the simple participant-spectator picture has to be viewed with some caution.

\section{A sampler of AGS results}

In the remainder of this second lecture, I discuss results obtained with heavy ions at the AGS accelerator of Brookhaven National Laboratory. I will highlight the mature results that have been obtained with silicon beams at $14.6 \mathrm{GeV} /$ nucleon as well as preliminary results obtained with recently available beams of $\mathrm{Au}$ ions at $11.6 \mathrm{GeV} /$ nucleon. Since I am not personally associated with any AGS experiment, I have relied heavily on information provided to me by some of my colleagues. In particular, I am grateful to Professor J. Stachel of the State University of New York at Stony Brook, who has provided me with copies of transparencies from a review talk that she presented recently. 
I have also made use of a recent article by Professor P. Braun-Munzinger of the same university [14].

The discussion of nuclear stopping in Section 2 above was based on measurements of transverse energy. A more direct determination of the degree of stopping is obtained from measured rapidity distributions of protons. As was pointed out, nuclear stopping is characterized by the rapidity loss of projectile protons or by the boosting of the protons to central rapidity. The distribution of protons from the reaction $\mathrm{Si}+\mathrm{Al}$ at 14.6 $\mathrm{GeV} /$ nucleon is shown in Fig. 7 [15]. The absence of a peak at the beam rapidity indicates that the degree of stopping is large. However, the width of the observed peak is larger than the width that would have been produced by a source involving complete stopping, followed by isotropic emission. It can be concluded that either the degree of stopping for this case is only in the $65 \%-70 \%$ range, or that the protons are initially fully stopped, and that their emission takes place subsequently from a longitudinally expanding source. In contrast, stopping at AGS energies is essentially complete for systems that involve at least one very heavy nucleus, such as lead. A compilation of proton rapidity distributions from central collisions of ${ }^{28} \mathrm{Si}+\mathrm{Pb}$ obtained by AGS experiments E802, E810, and E814 indicates that the agreement between the different experiments is good, that the stopping is consistent with $100 \%$, and that the observed results are well-described by standard event generators.

Full stopping and high baryon densities can also be deduced from recent measurements of transverse energy production in $\mathrm{Au}+\mathrm{Au}$ reactions at $11.4 \mathrm{GeV} /$ nucleon. $d E_{T} / d \eta$ distributions from experiment E814/E877 are shown in Fig. 8 [14]. Note that $E_{T}$ values produced per unit of pseudorapidity are about nine times larger in the $\mathrm{Au}+\mathrm{Au}$ case than in the $\mathrm{Si}+\mathrm{Al}$ case. Allowing for the difference in beam energy, the increase in $E_{T}$ density in the central rapidity region is nearly $50 \%$ greater than that expected from the available energy in the center-of-mass. Event generators that adequately describe transverse energy production indicate, for the $\mathrm{Au}+\mathrm{Au}$ case, an initial energy density of $\sim 1.5 \mathrm{GeV} / \mathrm{fm}^{3}$ and an initial baryon density of more than eight times that of normal nuclear matter.

It was pointed out in Section 2 that $d E_{T} / d \eta$ distributions at CERN energies have Gaussian shapes and that, consequently, the systematics of transverse energy production can be studied with reference to maximum values, averages, and widths of these distributions. From Fig 8 it can be seen that the distributions are also Gaussian at AGS energies. In Fig. 9 the Gaussian parameters are plotted for a number of reactions studied by the E814/E877 Collaboration as a function of collision centrality [16]. One interesting feature of these plots is that, with increasing centrality, the centroids shift to larger values of pseudorapidity in the $\mathrm{Au}+\mathrm{Al}$ case and to lower values in the $\mathrm{Si}+\mathrm{Pb}$ case relative to the symmetric $\mathrm{Si}+\mathrm{Al}$ and $\mathrm{Au}+\mathrm{Au}$ cases. The explanation lies in the forward-shift of the effective c.m. in the former case and in the backward-shift of the c.m. in the latter case relative to those of the symmetric systems. The Gaussian shape of the $E_{T}$ pseudorapidity distributions can be used to extrapolate the distributions to pseudorapidity regions where measurements have not been made. Thus estimates of 
the total $E_{T}$ (in the full solid angle) can be obtained. From such studies it has been concluded that a total of $18.5 \mathrm{GeV}$ of $E_{T}$ is produced per $\mathrm{GeV}$ in $\sqrt{s}$.

We next consider emission source sizes at freeze-out as deduced from pion-pion correlations. Interferometry studies have been introduced in Section 4 in the context of source sizes in the target fragmentation region. Two-pion correlation functions for both positive and negative pions from central $\mathrm{Si}+\mathrm{Pb}$ collisions at $14.6 \mathrm{GeV} /$ nucleon are shown in Fig. 10. As in the case discussed in Section 4, no reliable analytical expressions are available in this case, and the data were evaluated with reference to a theoretical model. Here, the Relativistic Quantum-Molecular Dynamics (RQMD) event generator [17] was used to calculate the correlation functions within the acceptance of the E814 spectrometer [18]. Excellent agreement was obtained between measured and calculated correlation functions. The analysis yielded a nearly spherical source with a transverse radius of 6.7 $\mathrm{fm}$ and a longitudinal radius, in the c.m. frame, of $5.0 \mathrm{fm}$. This corresponds to an rms radius of $8.3 \mathrm{fm}$. Such a large source radius can be compared to the $3.04-\mathrm{fm}$ rms radius of the Si nucleus, implying a transverse expansion of the system of about a factor of 3 . Under certain assumptions, we can conclude that the transverse expansion velocity is given by $\beta \approx 0.5$, resulting in an estimate for the collective expansion-time of about 10 $\mathrm{fm} / \mathrm{c}$.

Pion $p_{T}$ spectra were shown for CERN results in Section 3. Spectra presented as a function of the transverse mass, $m_{T}$, can be related to a system at equilibrium at a temperature of $T_{B}$ via the relationship $\sigma_{i n v .} \propto m_{T} \cdot \exp \left(-m_{T} / T_{B}\right)$, where $m_{T}=\sqrt{m^{2}+p_{T}^{2}}$. The Boltzmann shape is modified at low values of transverse momentum by pions originating from the decay of resonances at freeze-out. Analysis of this low- $p_{T}$ component provides us with information on the population of nucleon-excited states. A particularly instructive application of these considerations is provided by the $\Delta$-resonancethermometer analysis of pion spectra of the E814 Collaboration [14]. In Fig. 11 the $\pi^{-}$ yield divided by the Boltzmann spectrum fitted to $p_{T}$ values greater than $0.3 \mathrm{GeV} / \mathrm{c}$ is shown as a function of $m_{T}-m_{\pi}$, where $m_{\pi}$ is the pion mass. For $m_{T}-m_{\pi}$ values greater than 0.2 , the spectra follow a thermal shape, while at lower $m_{T}$ values, significant enhancements are observed. Also shown in Fig. 11 are predictions for the spectral shape based on two assumed contributions from $\Delta(1232)$ resonance excitation. Values of the ratio of pions originating from $\Delta$ decay to thermal (direct) pions between 0.4 and 0.6 bracket the observed results, leading to a value of 0.37 for $\frac{\Delta}{N+\Delta}$, where $N$ is the number of observed nucleons. The $\Delta(1232)$ resonance is also observed directly by the E814 Collaboration via invariant-mass reconstructions of $p \pi^{+}$combinations. In Fig. 12 results are shown for $\mathrm{Si}+\mathrm{Pb}$ central collisions at $14.6 \mathrm{GeV} /$ nucleon. The total $\Delta^{++}$multiplicity is $2.1 \pm 0.7$. The RQMD prediction for the same rapidity interval, $y=1.9-3.1$, is 1.8 , which is in reasonable agreement with the measured value. To obtain an estimate of the temperature from the above results, population probabilities of all nonstrange nucleon resonances with masses below $2 \mathrm{GeV}$ are considered. These are shown in Fig. 13 as a function of the temperature $T$ [14]. As expected, the $\Delta$ resonance is the dominant state. From the measured $\Delta$ to nucleon ratio of 0.37 , one can conclude on the basis of Fig. 13, that the temperature is $140 \mathrm{MeV}$ in the system under consideration. This value 
is consistent with a temperature of about $150 \mathrm{MeV}$ determined from direct analysis of pion spectra.

"Temperatures" determined from slopes of spectra of identified baryons or mesons vary depending on the species of the emitted particle. For example, $m_{T}$ spectra of protons and deuterons from central Au+Au collisions obtained by the E866 Collaboration, yield inverse slope parameters of $201 \pm 3 \mathrm{MeV}$ in the proton case and $250 \pm 15 \mathrm{MeV}$ in the deuteron case. The Boltzmann temperature deduced from the proton spectrum is 172 $\mathrm{MeV}$. These values compare to temperatures in the $150-\mathrm{MeV}$ range obtained from pion and kaon spectra. Preliminary inverse slope-parameter values for protons from central $\mathrm{Au}+\mathrm{Au}$ and $\mathrm{Si}+\mathrm{Al}$ collisions as a function of rapidity are shown in Fig. 14. The much flatter shape observed in the Au+Au case is probably due to a greater degree of overall stopping and thermalization.

In the final example of AGS results, I present results on $K^{+}$production from $\mathrm{Au}+\mathrm{Au}$ collisions [19]. The earliest results on strangeness enhancement were obtained by the E802 Collaboration [20]. An enhancement effect in the $K^{+}$yields was found relative to both pion and $K^{-}$production. In the rapidity range from 1.2 to 1.4 , the $K^{+} / \pi^{+}$ratios were found to be 0.078 in $\mathrm{p}+\mathrm{Be}, 0.125$ in $\mathrm{p}+\mathrm{Au}$, and 0.182 in $\mathrm{Si}+\mathrm{Au}$. The peaks of the $K^{+}$rapidity distributions were found to be at the same rapidities as those of the pions. In a study of $\mathrm{K}^{+} / \pi^{+}$ratios as a function of target mass and centrality, it was found that the enhancement effect was most pronounced in central $\mathrm{Si}+\mathrm{Au}$ collisions, that $\mathrm{Si}+\mathrm{Al}$ values were lower (presumably due to incomplete stopping effects), and that noncentral $\mathrm{Si}+\mathrm{Cu}$ values were similar to those of $\mathrm{Si}+\mathrm{Al}$. The $\mathrm{Au}+\mathrm{Au}$ result of the E802/E866 Collaboration is shown in Fig. 15 together with results for lighter systems. In the $\mathrm{Au}+\mathrm{Au}$ case, the value of $K^{+} / \pi^{+}$reaches about 0.25 .

In summary, it has been learned at the AGS that: interacting nuclei stop each other to a large extent (about $70 \%$ in the $\mathrm{Si}+\mathrm{Al}$ case and nearly $100 \%$ in cases involving $\mathrm{Pb}$ and $\mathrm{Au}$ nuclei); longitudinal and transverse expansions take place during the course of the reaction resulting in a large freeze-out radius; and the freeze-out temperatures are in the vicinity of $140 \mathrm{MeV}$.

\section{The Relativistic Heavy Ion Collider at the Brookhaven National Laboratory}

In this third lecture I present plans for experiments at the Relativistic Heavy Ion Collider (RHIC) under construction at the Brookhaven National Laboratory. The primary purpose of this large accelerator is the search for the quark-gluon plasma (QGP), which is a state of matter in which quarks and gluons are deconfined over a volume of nuclear dimensions. The fixed-target experiments at CERN's SPS and BNL's AGS that were discussed in the first two lectures also have as one of their goals the production of the QGP. However, as was pointed out in Section 2, the energy densities attained in the SPS and AGS energy regimes may not be sufficiently high for the phase transition to the QGP to take place. Some of the experimental results obtained thus far, such as the suppression of $J / \psi$ production in central nucleus-nucleus collisions, can be explained on 
the basis of QGP formation. Unfortunately, essentially all of the observations can also be explained by means of other effects, including those resulting from the creation of hot, dense hadronic matter. Thus, the principal value of the data obtained until now at the fixed-target experiments is to provide us with a basis of understanding of the general features of nucleus-nucleus reactions at these extreme energies. In late 1994 the last phase of fixed-target heavy-ion experiments at CERN will be initiated, with the availability of beams of lead ions with energies of $160 \mathrm{GeV} /$ nucleon. While it is possible that reactions with these very heavy projectiles will provide us with less ambiguous results regarding possible QGP formation, the focus of the community is shifting to the two collider projects: RHIC, a project dedicated to nucleus-nucleus studies, and the Large Hadron Collider (LHC), which is proposed at CERN primarily for particle-physics studies but which will also have the capability to accelerate heavy ions. One other point needs to be made. If the QGP is produced in fixed-target experiments, it will be formed in the relatively low-temperature and high-density region of the phase diagram. Its baryon content will be large, due to the presence of projectile and target baryons at all values of rapidity. On the other hand, in the collider regime, we anticipate that the QGP will be formed at a significantly higher temperature and that it will be nearly baryon-free in the central-rapidity region. We can expect major differences in the properties of the two types of plasma, and explanations of observed effects based on hadronic scenarios may not apply equally in the two cases.

RHIC has been a formal construction project since 1991, although the tunnel, the helium refrigeration system, and the experimental halls were constructed in the early $1980 \mathrm{~s}$ as part of the abandoned ISABELLE/CBA facility. Use will be made of the existing AGS, Booster, Tandem Van de Graaff, and proton linac accelerators. The site plan is shown in Fig. 16. Projectile energies will range from $100 \mathrm{GeV} /$ nucleon for $\mathrm{Au}$ ions to $250 \mathrm{GeV}$ for protons. The machine consists of two independent rings of superconducting magnets, making it possible to collide any combination of nuclear species including $\mathrm{p}+\mathrm{Au}$. The design luminosities are $2 \times 10^{26} / \mathrm{cm}^{2} / \mathrm{s}$ and $10^{31} / \mathrm{cm}^{2} / \mathrm{s}$ for $\mathrm{Au}+\mathrm{Au}$ at the maximum energy and for protons, respectively. These luminosities can be increased by an order of magnitude via upgrades that are already under consideration. The installation of dipoles at RHIC is currently underway and will take about two years to complete. Routine operation is expected to begin in the spring of 1999 .

There are two major experiments currently under construction, PHENIX and STAR. These are described below. In addition, two smaller experiments, BRAHMS and PHOBOS, are under active consideration, but will not be discussed here. Since I am personally associated with PHENIX and since this detector makes use of "penetrating probes" such as leptons and photons which are well-matched to studies of early reaction stages and of possible QGP formation, this experiment is emphasized in the remainder of this lecture. 


\section{The PHENIX experiment at RHIC}

The primary goal of PHENIX is to detect the QGP and to study its properties by performing systematic investigations of leptonic, photonic, and hadronic signatures. The design philosophy was to provide the capability of carrying out simultaneous studies of as many potential signatures of QGP formation as possible and to enable searches for correlated changes in several observables as a function of global parameters such as charged-particle multiplicity or energy density. Studies of both A+A and p+A reactions are envisaged. High resolution and good particle-identification capabilities are stressed. Physics topics and possible QGP signatures to be studied include the following:

a. Debye-screening of parton interactions due to large parton densities, leading to the "suppression" of the formation of heavy vector mesons such as the $J / \psi$, the $\psi^{\prime}$, the $\Upsilon$, and the $\Upsilon^{\prime}$;

b. chiral symmetry restoration which may result in changes of the mass and width of mesons such as the $\phi$;

c. thermal radiation from the QGP resulting in large emission probabilities of direct photons and of lepton pairs with transverse masses in the range of $1-4 \mathrm{GeV} / \mathrm{c}^{2}$;

d. first-order phase transition characterized by a large latent heat as determined, for example, from the variation of the transverse momentum of produced particles as a function of entropy density;

e. second-order phase transition as signaled by fluctuation phenomena such as nonGaussian distributions of neutral-to-total pion ratios.

Studies of strangeness and charm production, of jet-quenching, and of space-time evolution will also be pursued. Since some of the QGP signatures may involve rare processes and small effects, PHENIX is designed to be able to operate at the highest luminosities expected at RHIC.

An isometric view of the PHENIX detector is shown in Fig. 17. The detector consists essentially of two separate spectrometers: a two-arm electron-photon spectrometer located at midrapidity and a muon spectrometer located in the forward-rapidity region. The rapidity and azimuthal acceptances of the three spectrometer arms are shown in Fig. 18. In a limited region (also shown in Fig. 18) of one of the electron-photon arms, it is possible to identify hadrons by means of a time-of-flight system. Since a large range of momentum coverage is required for electron detection $(150 \mathrm{MeV} / \mathrm{c}-4 \mathrm{GeV} / \mathrm{c})$, several subsystems are required for adequate pion rejection over the entire dynamic range, including ring-imaging Cherenkov detectors and time-expansion chambers. While both electron-photon arms feature full electromagnetic calorimeter coverage, two different technologies are used: homogeneous lead-glass detectors and lead-scintillator sampling calorimeters. The array of lead-glass modules is installed behind the time-of-flight detector. The electron spectrometers are installed inside an axial-field magnet, while the 
muon spectrometer has its own "lampshade" magnet consisting of a central "piston" and of eight sheets of steel which make up the lampshade structure. The beam line is located along the central axis of the piston, which also supports the magnet coil at its base.

The design luminosity of RHIC is relatively low. However, this is somewhat compensated by the fact that production rates for massive objects, such as the $J / \psi$ and $\Upsilon$ mesons, scale approximately with $A^{1.8-1.2}$, where $A$ is the mass of the colliding nuclei. This results in reasonable expected counting rates as shown in Table 1.

Table 1. Typical yields per RHIC-year for various species into the PHENIX muon acceptance.

\begin{tabular}{lrr}
\hline & $\begin{array}{c}\mathrm{Au}+\mathrm{Au} \\
(\text { Central*) }\end{array}$ & $\begin{array}{c}\mathrm{p}+\mathrm{Au} \\
\text { (Min. bias) }\end{array}$ \\
\hline$\rho \rightarrow \mu^{+} \mu^{-}$ & $309 \mathrm{~K}$ & $920 \mathrm{~K}$ \\
$\phi \rightarrow \mu^{+} \mu^{-}$ & $139 \mathrm{~K}$ & $500 \mathrm{~K}$ \\
$J / \psi \rightarrow \mu^{+} \mu^{-}$ & $390 \mathrm{~K}$ & $1060 \mathrm{~K}$ \\
$\psi^{\prime} \rightarrow \mu^{+} \mu^{-}$ & $5.7 \mathrm{~K}$ & $15 \mathrm{~K}$ \\
$\Upsilon \rightarrow \mu^{+} \mu^{-}$ & $1.2 \mathrm{~K}$ & $1.4 \mathrm{~K}$ \\
$\Upsilon^{\prime} \rightarrow \mu^{+} \mu^{-}$ & $0.12 \mathrm{~K}$ & $0.14 \mathrm{~K}$ \\
Drell-Yan $\rightarrow \mu^{+} \mu^{-}$at $3 \mathrm{GeV}$ & $15 \mathrm{~K}$ & $26 \mathrm{~K}$ \\
$D \bar{D} \rightarrow \mu^{+} \mu^{-}$at $3 \mathrm{GeV}$ & $26 \mathrm{~K}$ & $73 \mathrm{~K}$ \\
$D \bar{D} \rightarrow e-\mu$ & $140 \mathrm{~K}$ & $370 \mathrm{~K}$ \\
\hline
\end{tabular}

${ }^{*} \mathrm{Au}+\mathrm{Au}$ central collisions correspond to $1 \%$ most central collisions for $2 \times 10^{27}$ luminosity, or to top $10 \%$ for $2 \times 10^{26}$ ("Blue Book") luminosity.

The Drell-Yan and $D \bar{D}$ yields are quoted per $\mathrm{GeV} / \mathrm{c}^{2}$. Yields into the electron arms are 3-10 times smaller due to acceptance and solid angle.

Simulated spectra of electrons and muons are shown in Figs. 19 and 20, respectively, for Au+Au collisions at standard design luminosity. Note that the $J / \psi$ and the $\psi^{\prime}$ in the electron spectrum and the $\Upsilon$ in the muon spectrum appear in regions that are essentially background-free. As can be noted from Table 1, the expected production rate for the $\Upsilon^{\prime}$ is only 120 events per year. Consequently, the higher states of the $\Upsilon$ are not seen in the one-month spectrum of Fig. 20. However, the resolution of the PHENIX muon spectrometer is adequate for the determination of yields of the higher $\Upsilon$ states given a sufficiently long running period.

\section{The STAR experiment at RHIC}

The emphasis of STAR (Solenoidal Tracker at RHIC) is on the measurement and correlation of global observables on an event-by-event basis and on the use of the hard- 
scattering of partons to probe properties of the QGP or of high-density hadronic matter. To accomplish these objectives, full track reconstruction and particle identification will be carried out for most charged particles emitted in any given collision in the two central units of rapidity, where the particle rapidity density is expected to be about 1000 . The detector consists of a time-projection chamber (TPC) located inside a $0.5-\mathrm{T}$ solenoidal magnetic field and surrounded by a time-of-flight barrel and an electromagnetic calorimeter. A silicon-drift detector will be located at the center of the TPC, and additional, external TPCs will cover regions of high rapidity. The track density that the detector has to be capable of handling over the entire detector volume is similar to that which is produced in a small region when a jet fragments into hadrons. Particle identification capability will be provided for pions, kaons, and protons at rapidities below 1 , and momentum spectra will be obtained for $\pi^{ \pm}, K^{ \pm}, K_{s}^{0}, p, \bar{p}, d, \bar{d}, \phi, \Lambda / \bar{\Lambda}, \Xi^{+}, \Xi^{-}$, and $\Omega^{-}$.

STAR intends to study a large range of physics topics including the following:

a. production of identified multistrange hyperons and anti-hyperons which may be sensitive to deconfinement and to attained temperatures as a consequence of strangeness enhancement and of required antistrange quark formation;

b. cross sections for jet, photon, photon-jet, and jet-jet production which may provide information on the propagation of partons in a deconfined QCD phase;

c. momentum correlations in single events leading to the determination of event shapes which may provide an indication of very rapid cooling of the reaction volume such as may be expected as a consequence of a first-order phase transition;

d. fluctuations in single events related to possible second-order phase transitions;

e. spectral shape parameters of a large range of identified particles which are related to the temperatures attained in individual collisions.

In conclusion, I have indicated that the RHIC project is well underway, and that the two major experiments planned for the facility, PHENIX and STAR, are being implemented with broad capabilities to address future exciting physics issues. A strong argument can be made that European groups should join the RHIC effort even with the advent of the LHC project at CERN. Aside from covering different energy regions, the two projects are shifted in time by over five years relative to each other, and RHIC is a machine dedicated to nucleus-nucleus studies, while the LHC will be available only on a limited basis. 


\section{References}

[1] R. Albrecht et al., WA80 Collaboration, Phys. Rev. C 44 (1991) 2736.

[2] R. Santo, WA80 Collaboration, Nucl. Phys. A566 (1994) 61c.

[3] T. C. Awes et al., WA80 Collaboration, paper submitted to Zeitschrift für Physik $C$ (July 1994).

[4] G. R. Young et al., Nucl. Instrum. Methods Phys. Res. A279 (1989) 503.

[5] T. C. Awes et al., Nucl. Instrum. Methods Phys. Res. A279 (1989) 479.

[6] C.-Y. Wong and Z.-D. Lu, Phys. Rev. D 39 (1989) 2606.

[7] J. D. Bjorken, Phys. Rev. D 27 (1983) 140.

[8] R. Albrecht et al., WA80 Collaboration, Z. Phys. C 51 (1991) 1.

[9] Reference 2 above gives a list of references for the hadron-hadron and hadronnucleus data depicted in Fig. 6.

[10] M. Bourquin and J. Gaillard, Nucl. Phys. B114 (1976) 334.

[11] D. K. Srivastava and B. Sinha, Phys. Rev. Lett. 73 (1994) 2421.

[12] E. V. Shuryak and L. Xiong, Phys. Lett. B 333 (1994) 316.

[13] S. Pratt, private communication, 1992.

[14] P. Braun-Munzinger, in Proceedings, NATO Advanced Study Institute on Hot and Dense Nuclear Matter, Bodrum, Turkey, Oct. 1993, Plenum Press, New York, 1994.

[15] J. Barrette et al., E814 Collaboration, Z. Phys. C 59 (1993) 211.

[16] J. Barrette et al., E814/E877 Collaboration, Phys. Rev. Lett. 70 (1993) 2996.

[17] H. Sorge, H. Stöcker, and W. Greiner, Ann. Phys. (NY) 192 (1989) 266; A. Jahns, H. Sorge, H. Stöcker, and W. Greiner, Z. Phys. A 341 (1992) 243.

[18] J. Barrette et al., E814 Collaboration, Phys. Lett. B 333 (1994) 33.

[19] M. Gonin for the E802/E866 Collaboration, in Proceedings, International Nuclear Physics Conference, Wiesbaden, Germany, 1992.

[20] T. Abbott et al., E802 Collaboration, Z. Phys. C 38 (1988) 135; T. Abbott et al., Phys. Rev. Lett. 66 (1991) 1567. 


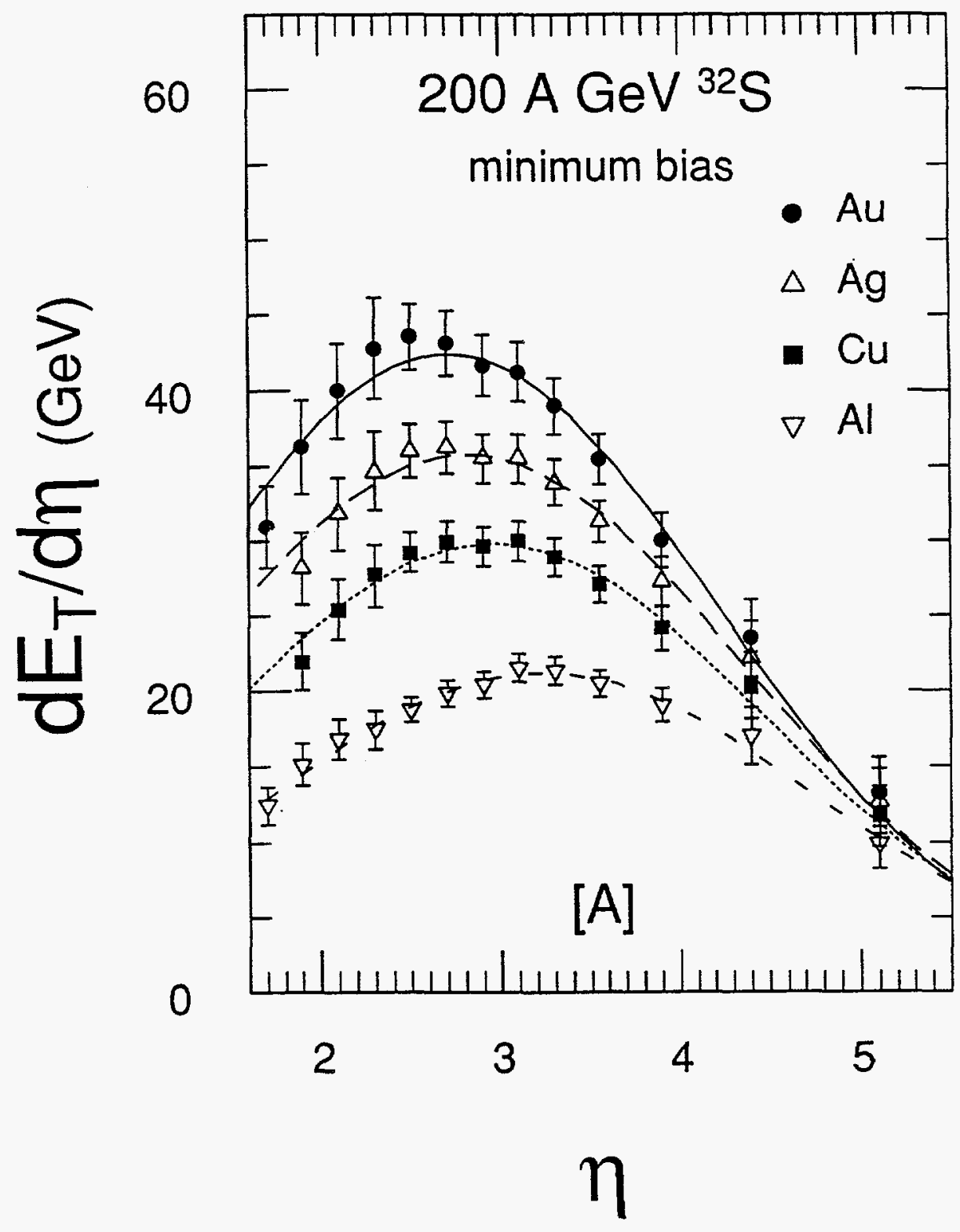

Figure 1. Target dependence of $\frac{d E_{T}}{d \eta}$ in $200 \mathrm{~A} \mathrm{GeV}{ }^{32} \mathrm{~S}$-induced minimum bias collisions. Figure from Ref. 1. 


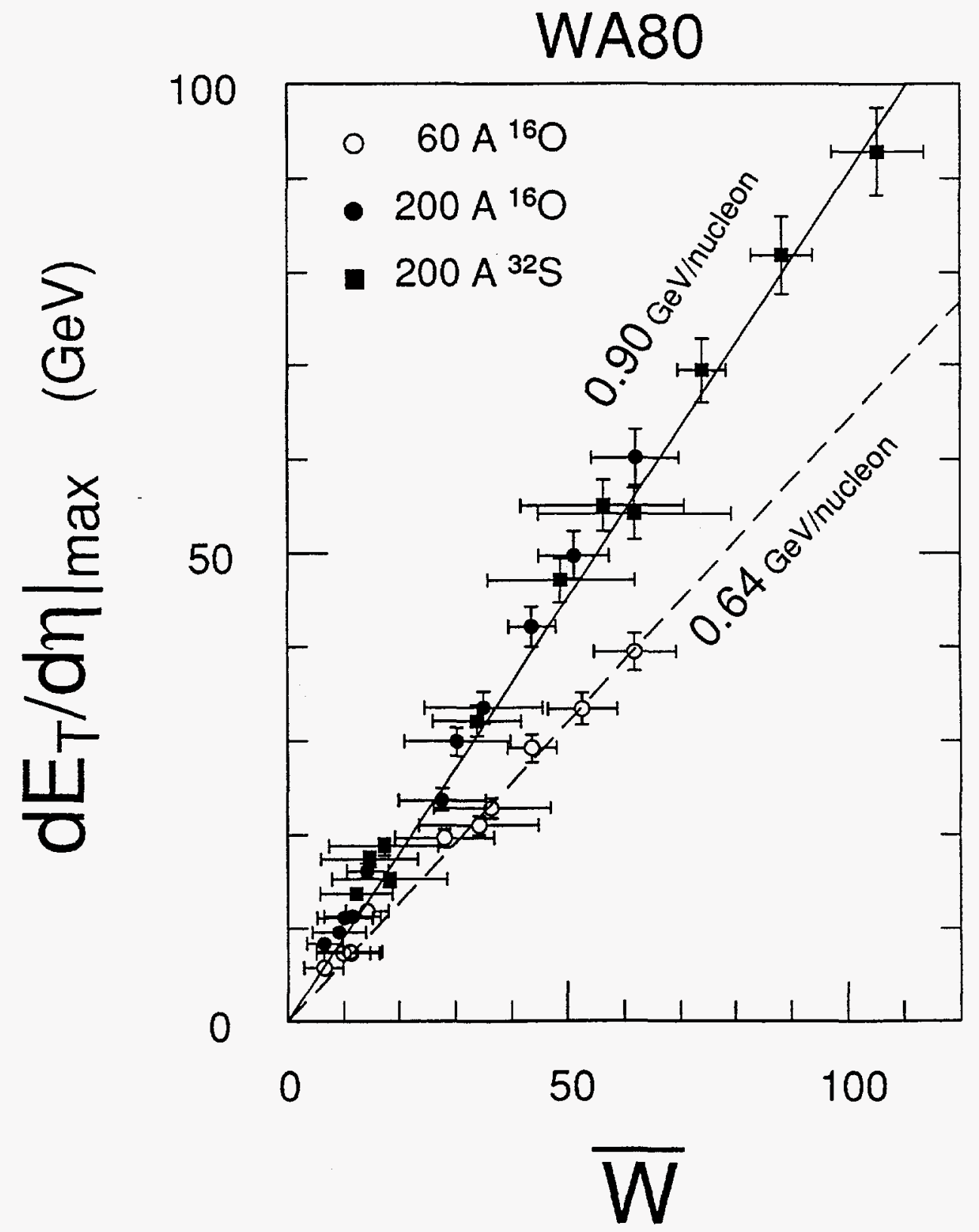

Figure 2. Maximum values of $\left.\frac{d E_{T}}{d \eta}\right|_{m a x}$ distributions as a function of the average total number of participants, $\bar{W}$. The straight lines indicate linear fits to the data, constrained to pass through the origin, at $60 \mathrm{~A}$ and $200 \mathrm{~A} \mathrm{GeV}$. The respective slope parameters are indicated. Figure from Ref. 1. 


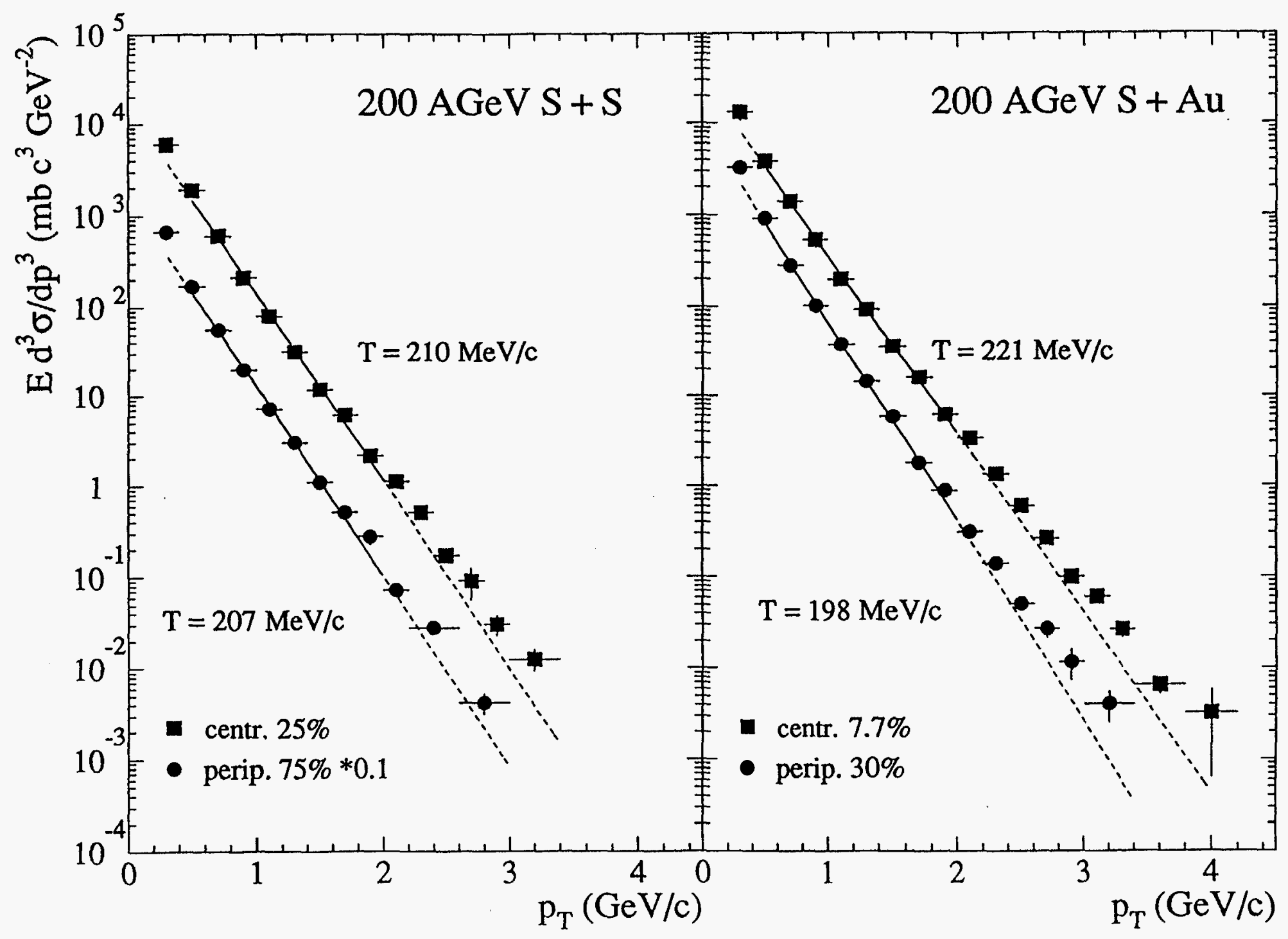

Figure 3. $\pi^{0}$ transverse momentum spectra for peripheral and central collisions of $S$ nuclei with $\mathrm{S}$ and $\mathrm{Au}$ nuclei at $200 \mathrm{GeV} /$ nucleon. The curves represent exponential fits in the transverse momentum range from 0.5 to $2.0 \mathrm{GeV} / \mathrm{c}$, with slope parameters, $T$, as indicated. Figure from Ref. 2. 


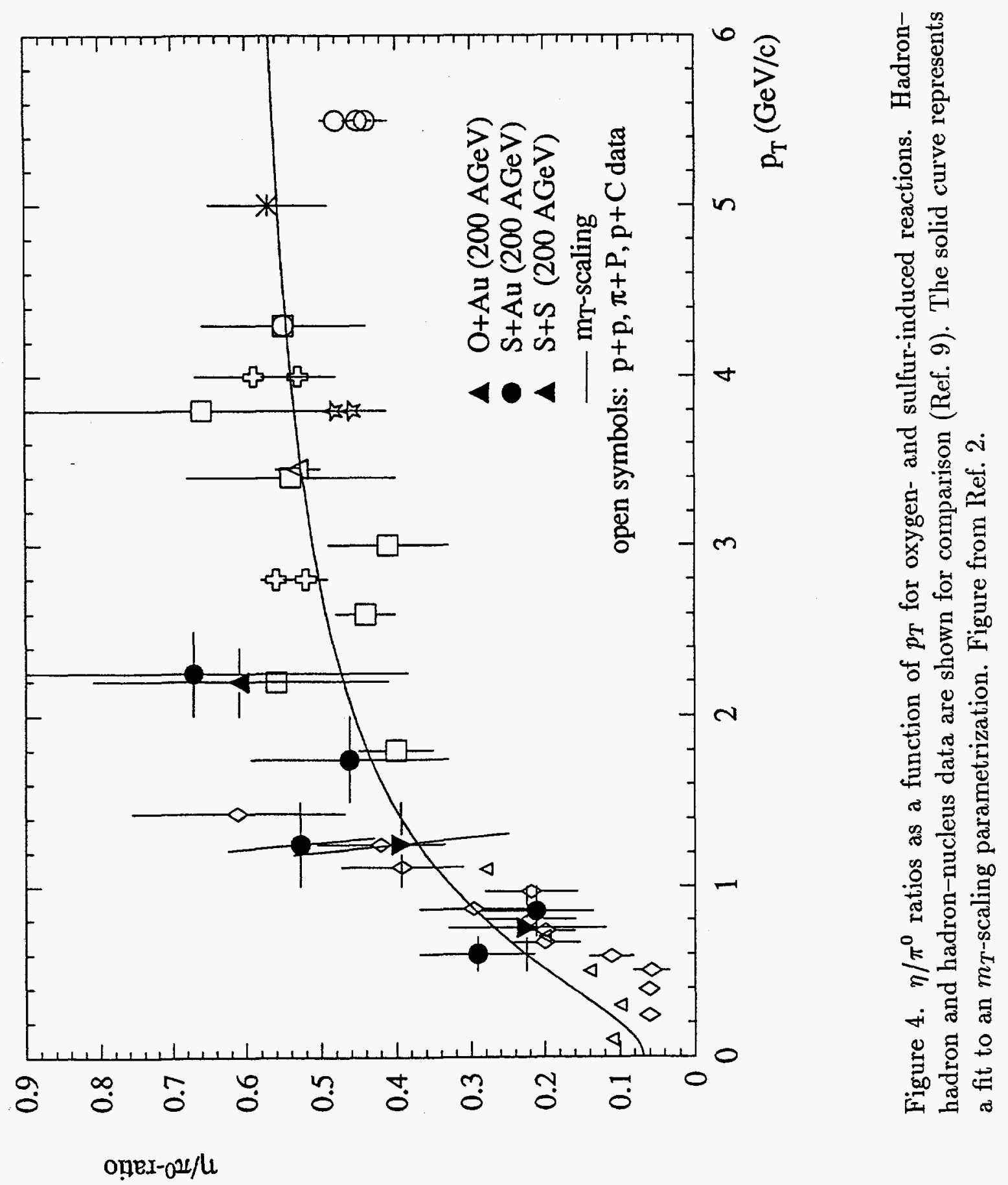




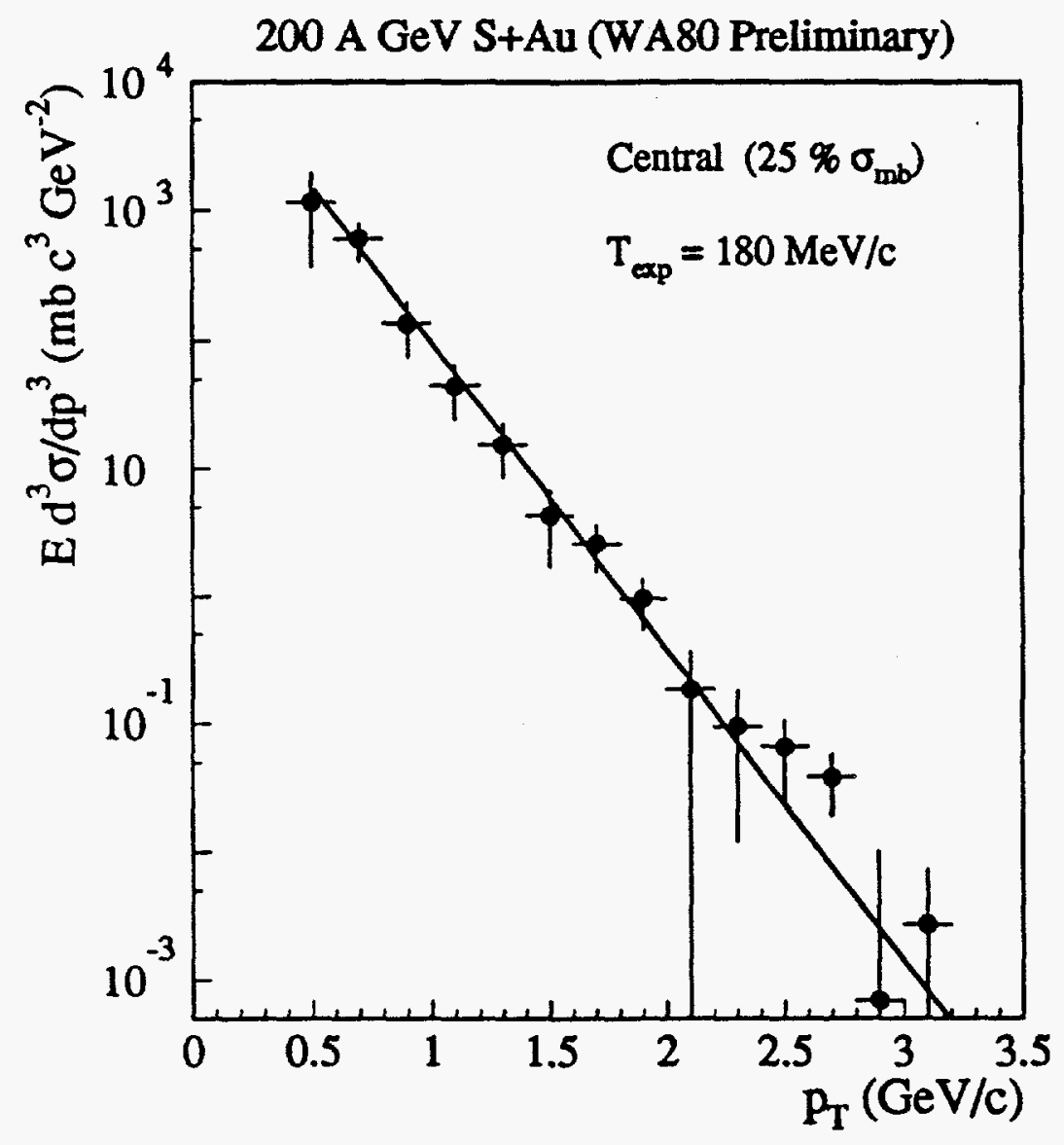

Figure 5. The excess photon transverse momentum distribution for the $25 \% \sigma_{\min }$ bias $(900 \mathrm{mb})$ most central interactions of $200 \mathrm{~A} \mathrm{GeV} \mathrm{S}+\mathrm{Au}$. The errors shown include all statistical and estimated systematic errors of the experimental and background simulation results. 


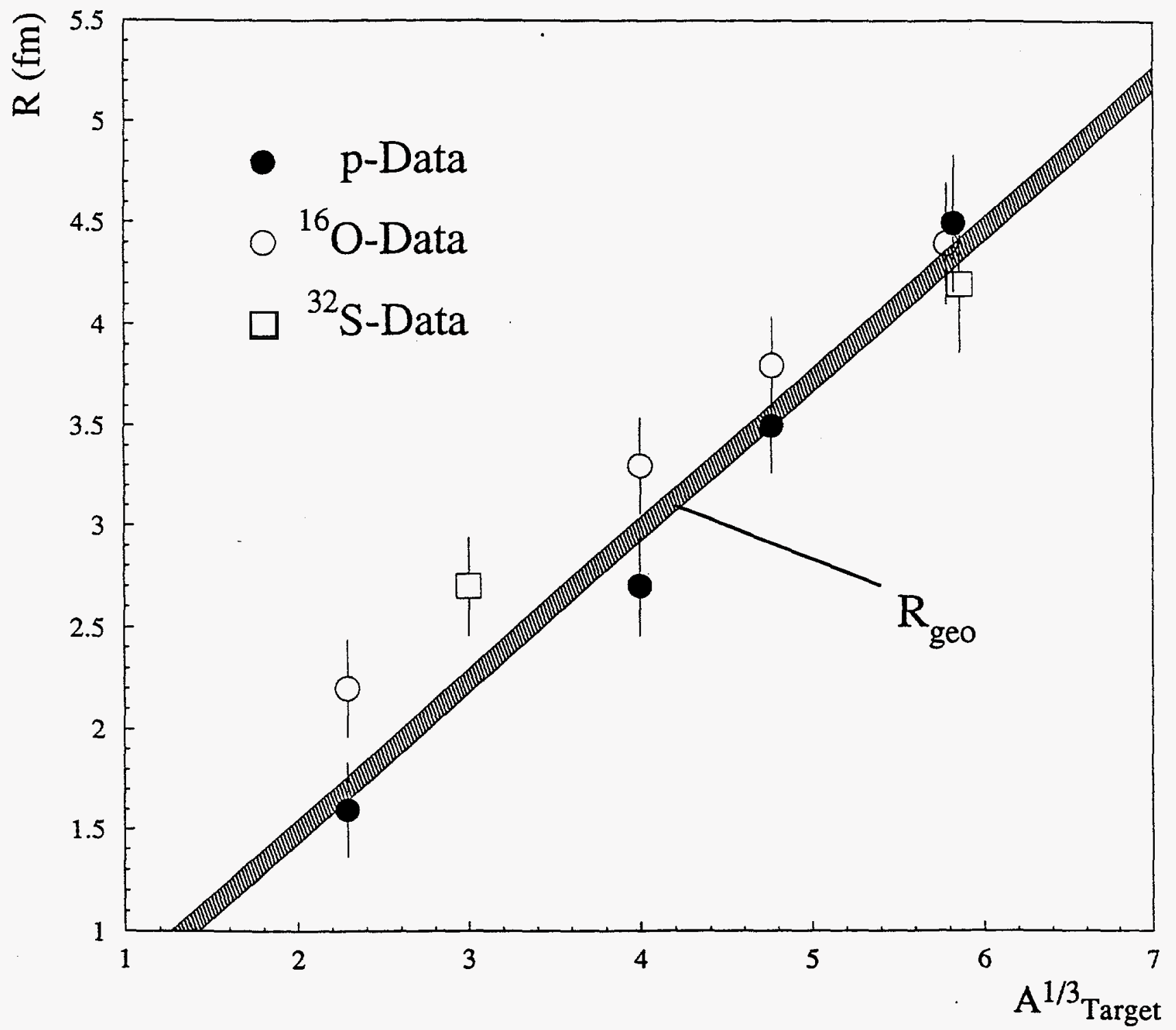

Figure 6. Target-dependence of radii, $R$, extracted from proton-proton correlation data, as a function of target $A^{1 / 3}$. The hatched band labeled $R_{g e o}$ represents geometric radii of target nuclei. 


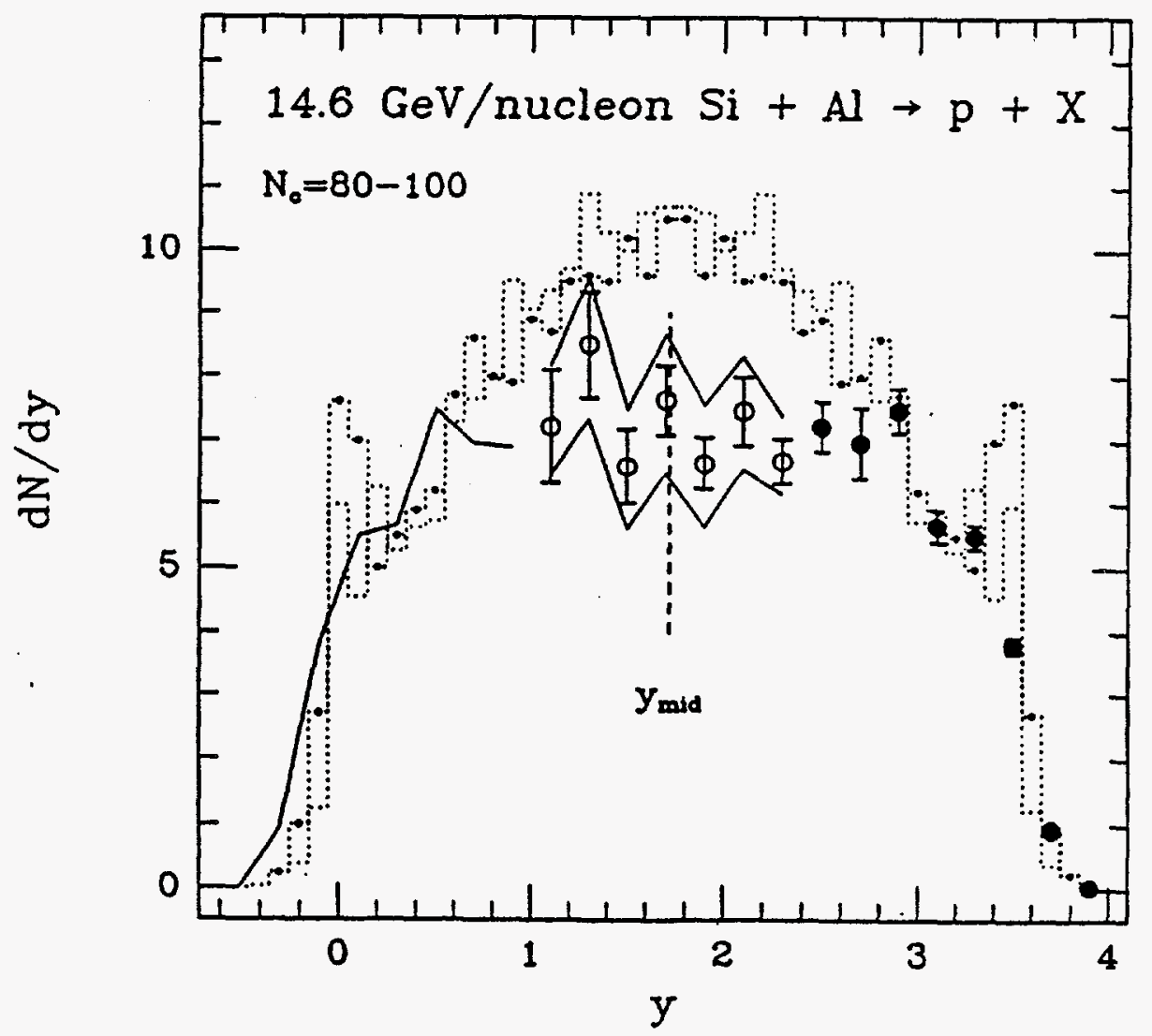

Figure 7. Proton rapidity distributions for central $\mathrm{Si}+\mathrm{Al}$ collisions $\left(\sigma / \sigma_{\text {geo }}=0.2 \%\right)$. The solid line for $y \leq 1$ represents data reflected about midrapidity; the two separated lines indicate the upper and lower limits due to extrapolation. The histograms with and without solid dots are different model predictions. Figure from Ref. 15. 


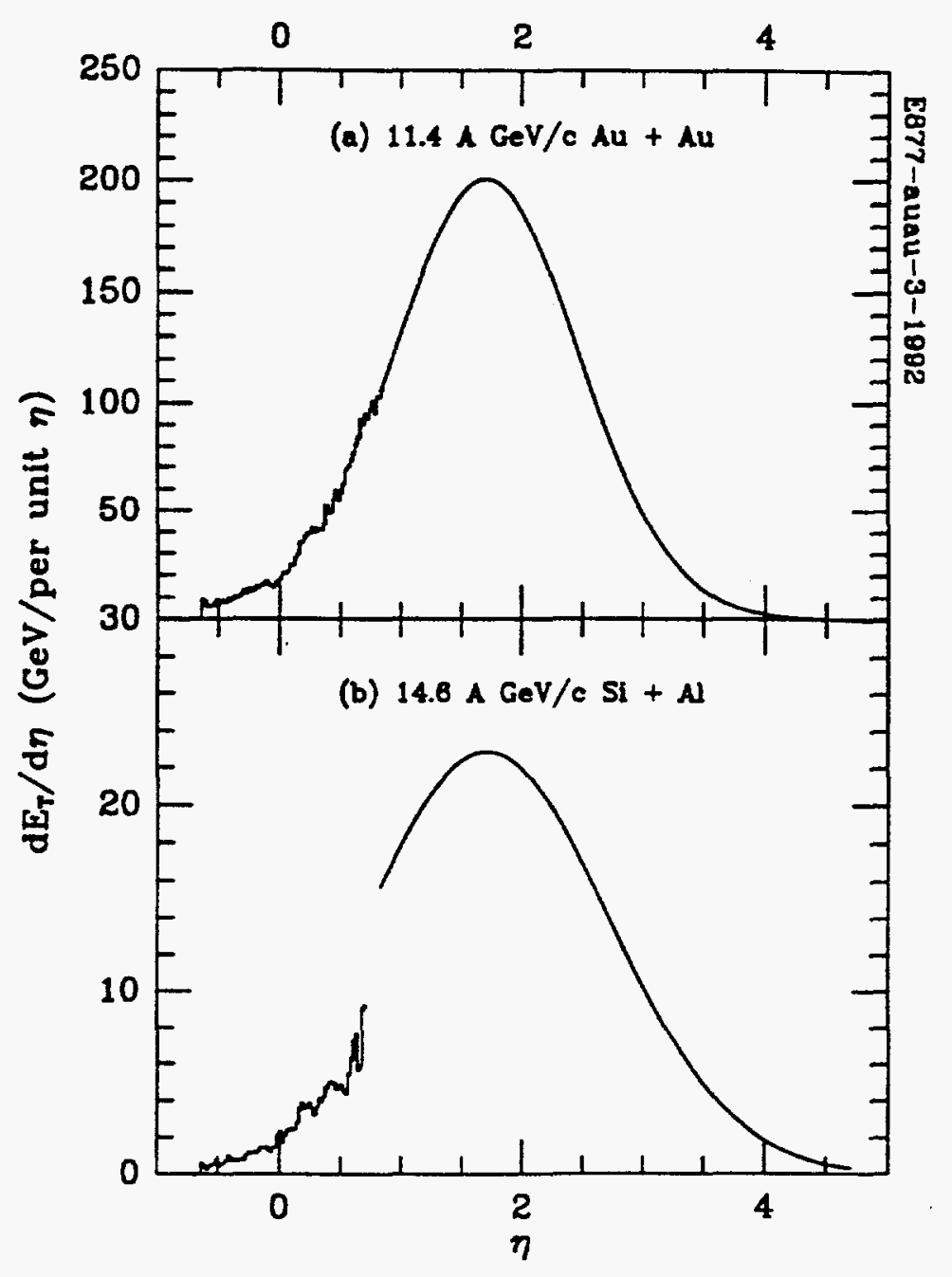

Figure 8. Experimental $d E_{T} / d \eta$ distributions from central $\mathrm{Si}+\mathrm{Al}$ and $\mathrm{Au}+\mathrm{Au}$ collisions. Figure from Ref. 14. 


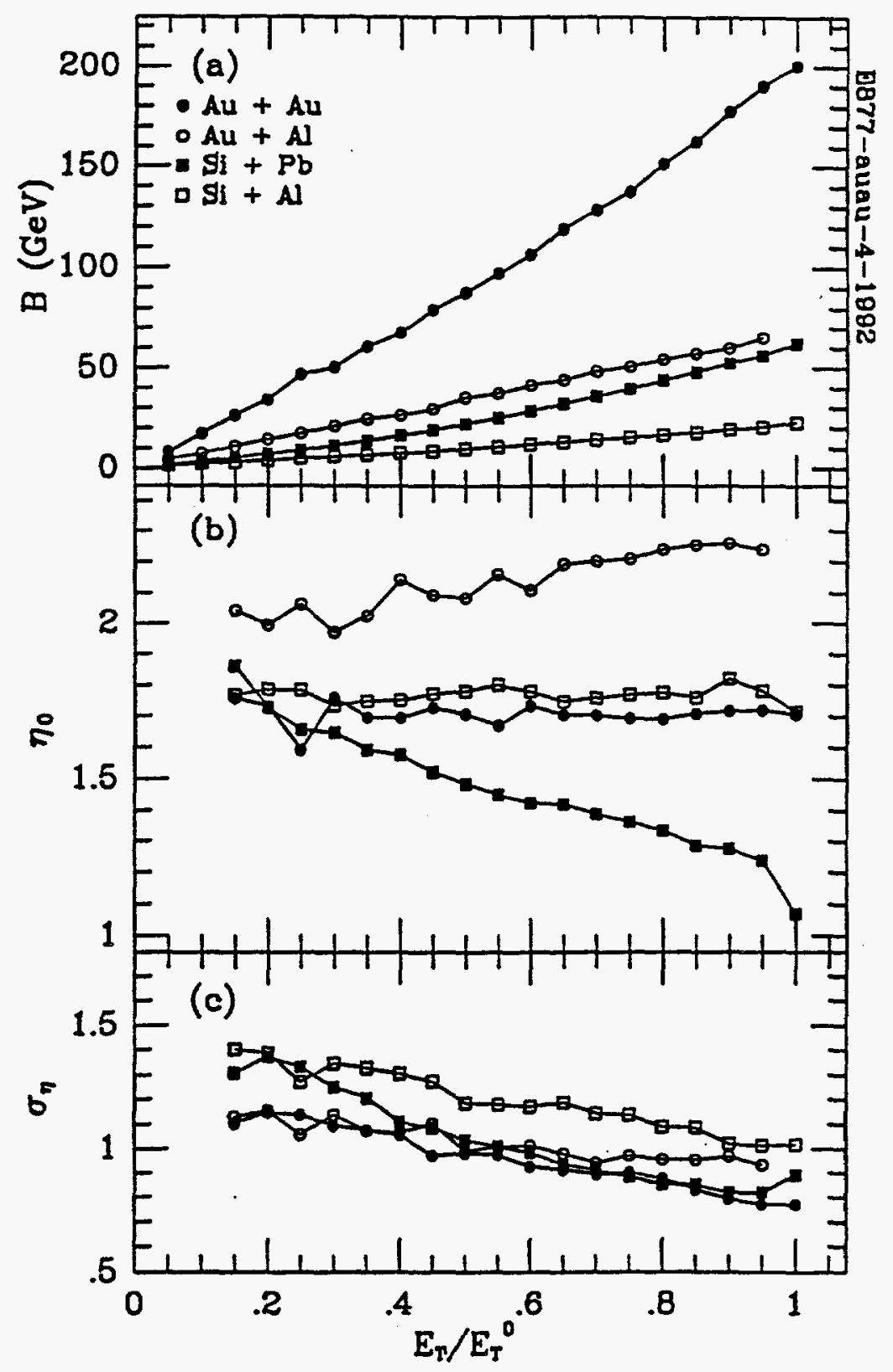

Figure 9. Parameters for the Gaussian functions representing measured $d E_{T} / d \eta$ distributions as a function of the ratio of $E_{T}$ to $E_{T}^{0}$, where $E_{T}^{0}$ is the mean $E_{T}$ for a zero-impact-parameter collision. $E_{T} / E_{T}^{0}$ is a measure of event centrality. B represents the maximum value; $\eta_{0}$ the average; and $\sigma_{\eta}$ the width. Figure from Ref. 16. 


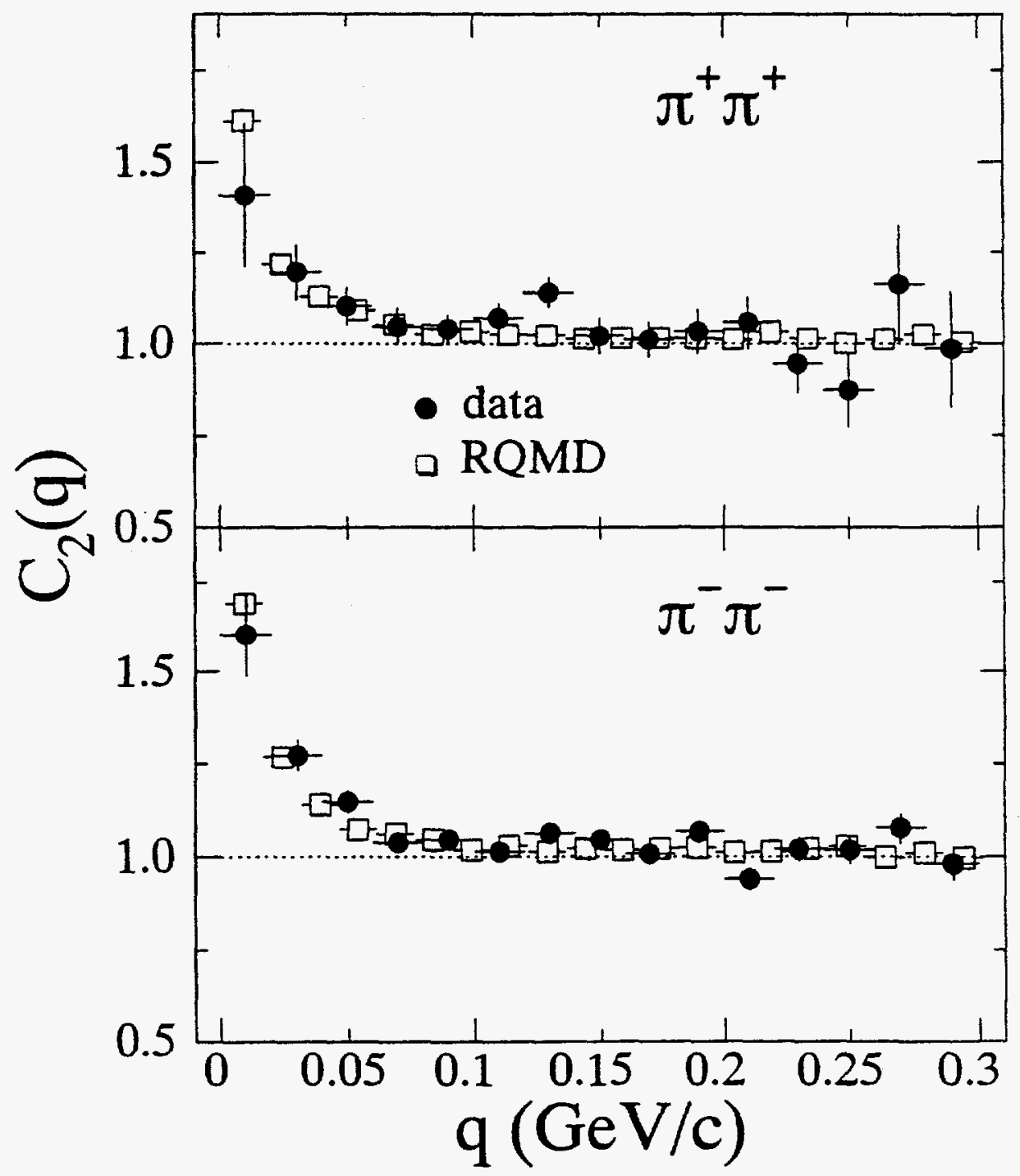

Figure 10. Experimental two-pion correlation function and the corresponding correlation function constructed from RQMD [17] events. Figure from Ref. 18. 


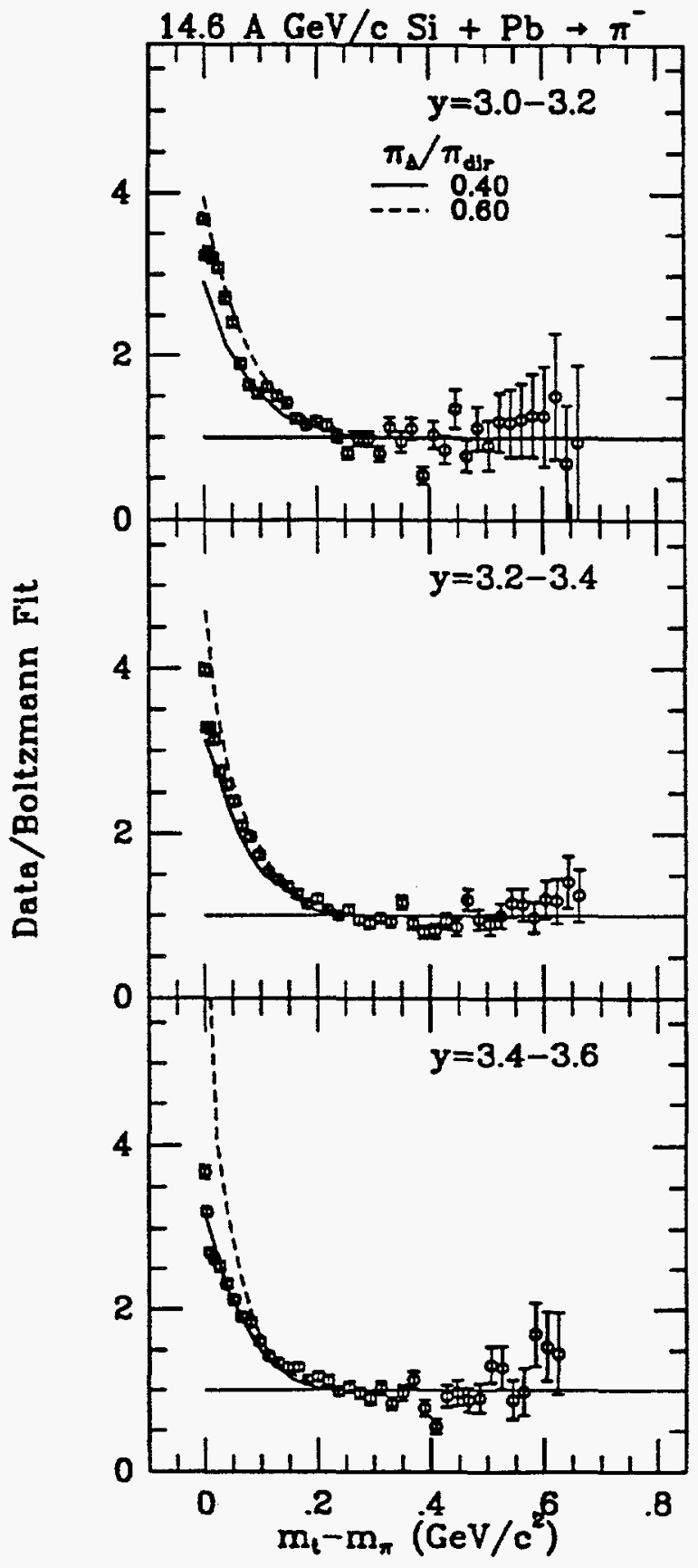

Figure 11. Experimental $\pi^{-}$spectra normalized to a Boltzmann distribution fitted to the data for $p_{T} \geq 0.3 \mathrm{GeV} / \mathrm{c}$. Solid and dashed lines: thermal model with different fractions of $\Delta$ decay versus direct pions. Figure from Ref. 14. 


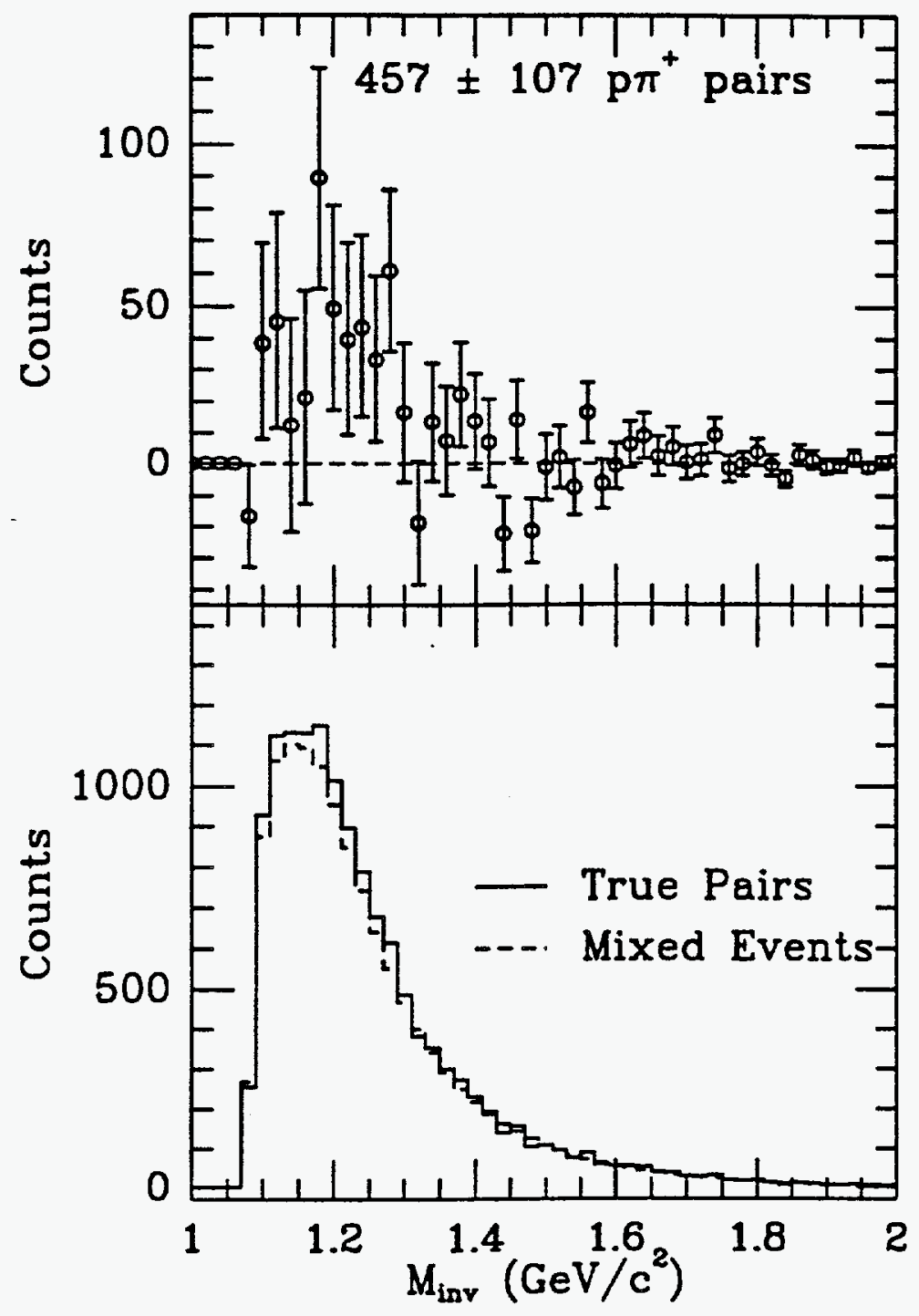

Figure 12. Reconstruction of the $\Delta^{++}$resonance for central $\mathrm{Si}-\mathrm{Pb}$ collisions at 14.6 $\mathrm{GeV} /$ nucleon. Figure from Ref. 14. 


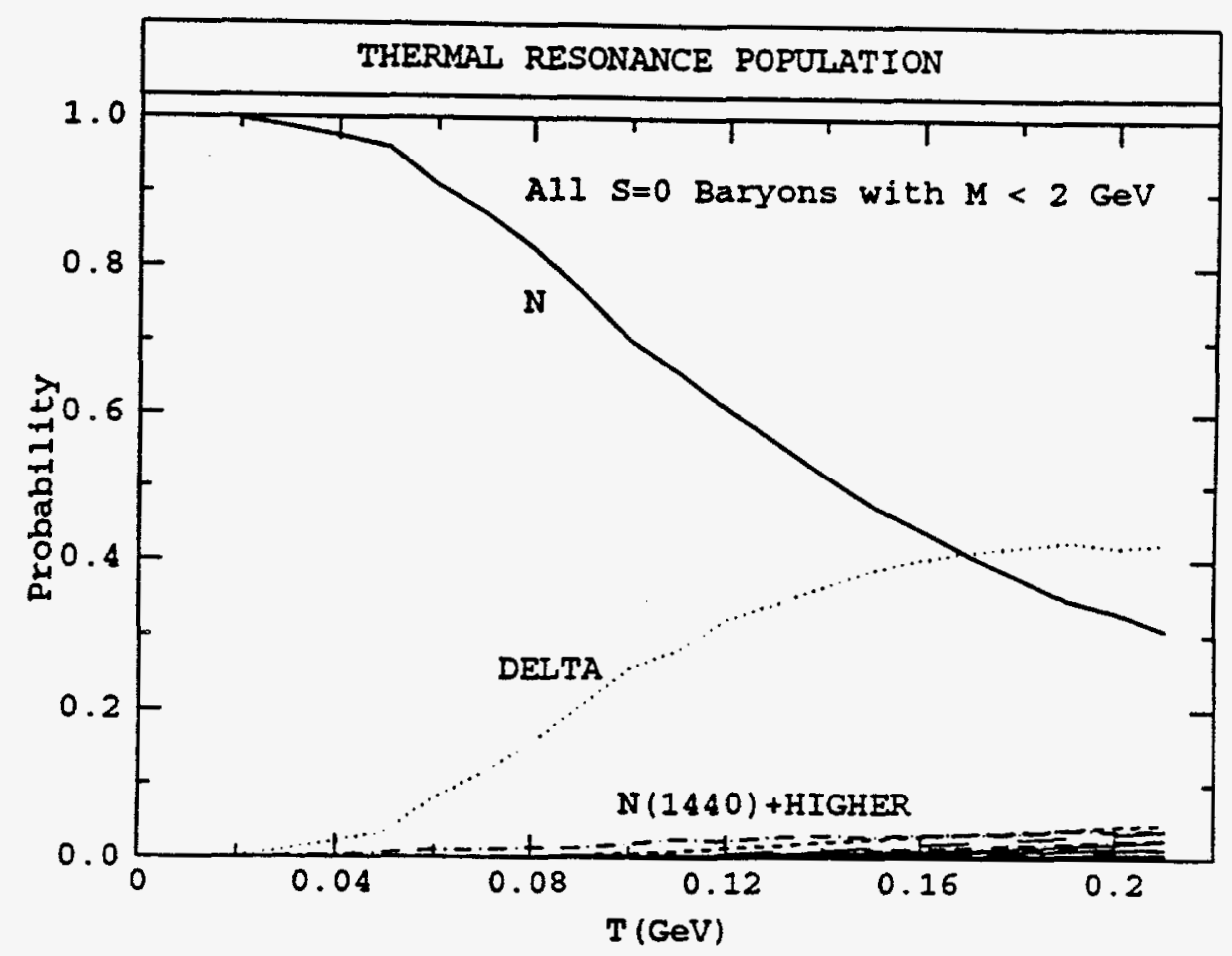

Figure 13. Population of nucleon resonances as a function of the temperature. The widths of the resonances are included in the calculation. Figure from Ref. 14. 


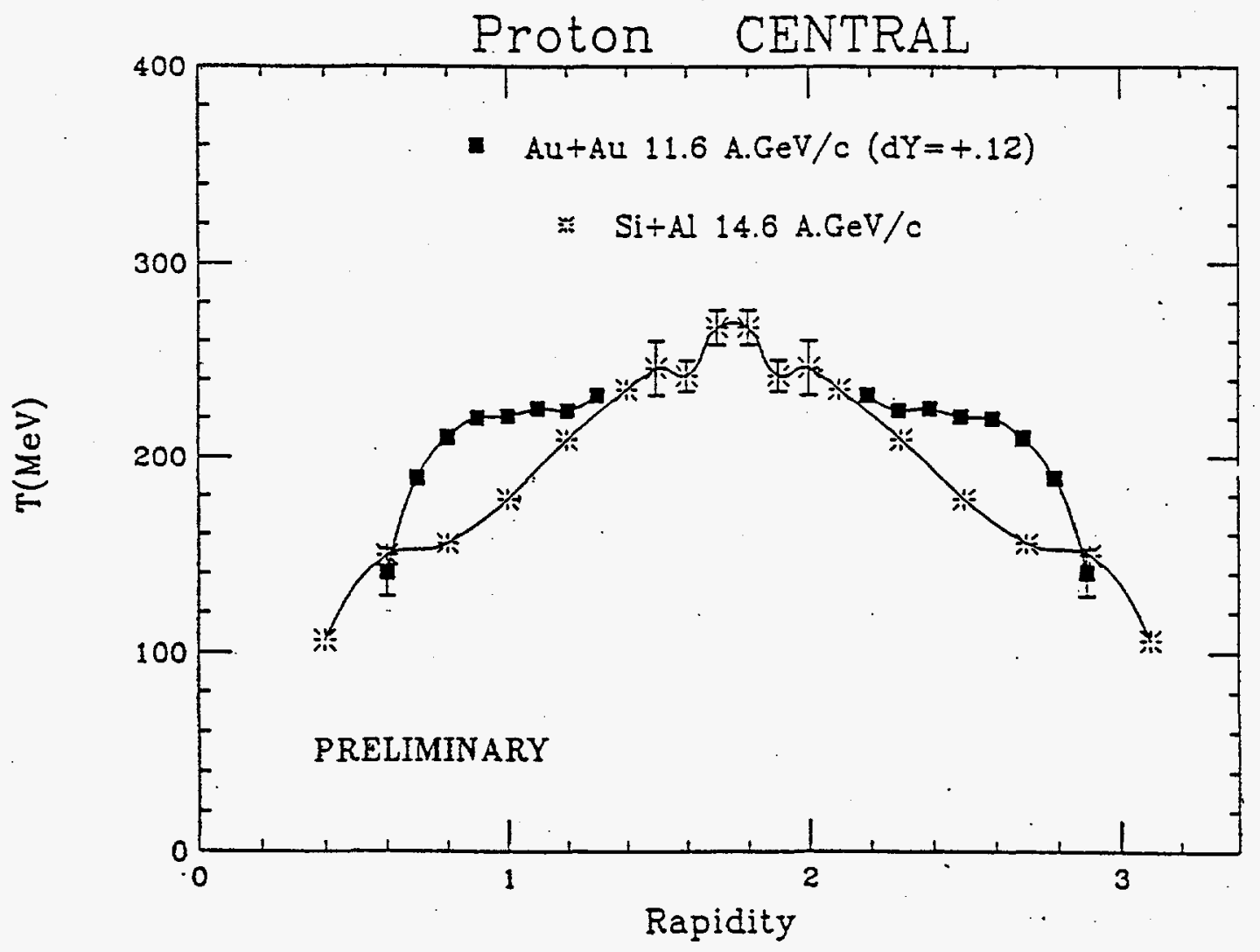

Figure 14. Inverse slope parameter values versus rapidity for protons from $A u+A u$ central collisions (AGS experiment E866) and from $\mathrm{Si}+\mathrm{Al}$ central collisions (AGS experiment E802). 


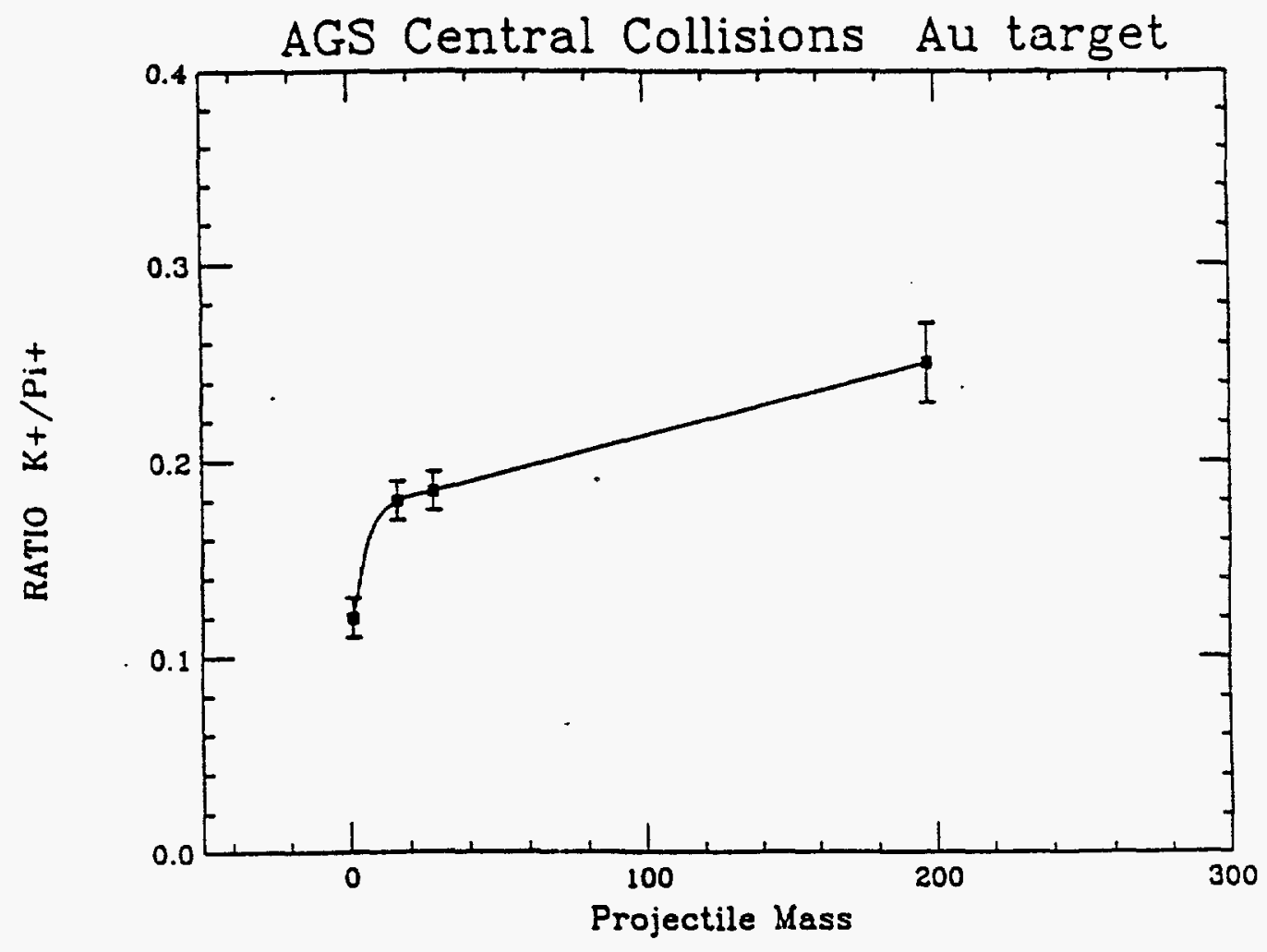

Figure $15 . K^{+} / \pi^{+}$ratios as a function of projectile mass for $p+\mathrm{Au}, \mathrm{O}+\mathrm{Au}, \mathrm{Si}+\mathrm{Au}$, and Au+Au obtained by the E802 and E866 Collaborations. Figure from Ref. 19. 


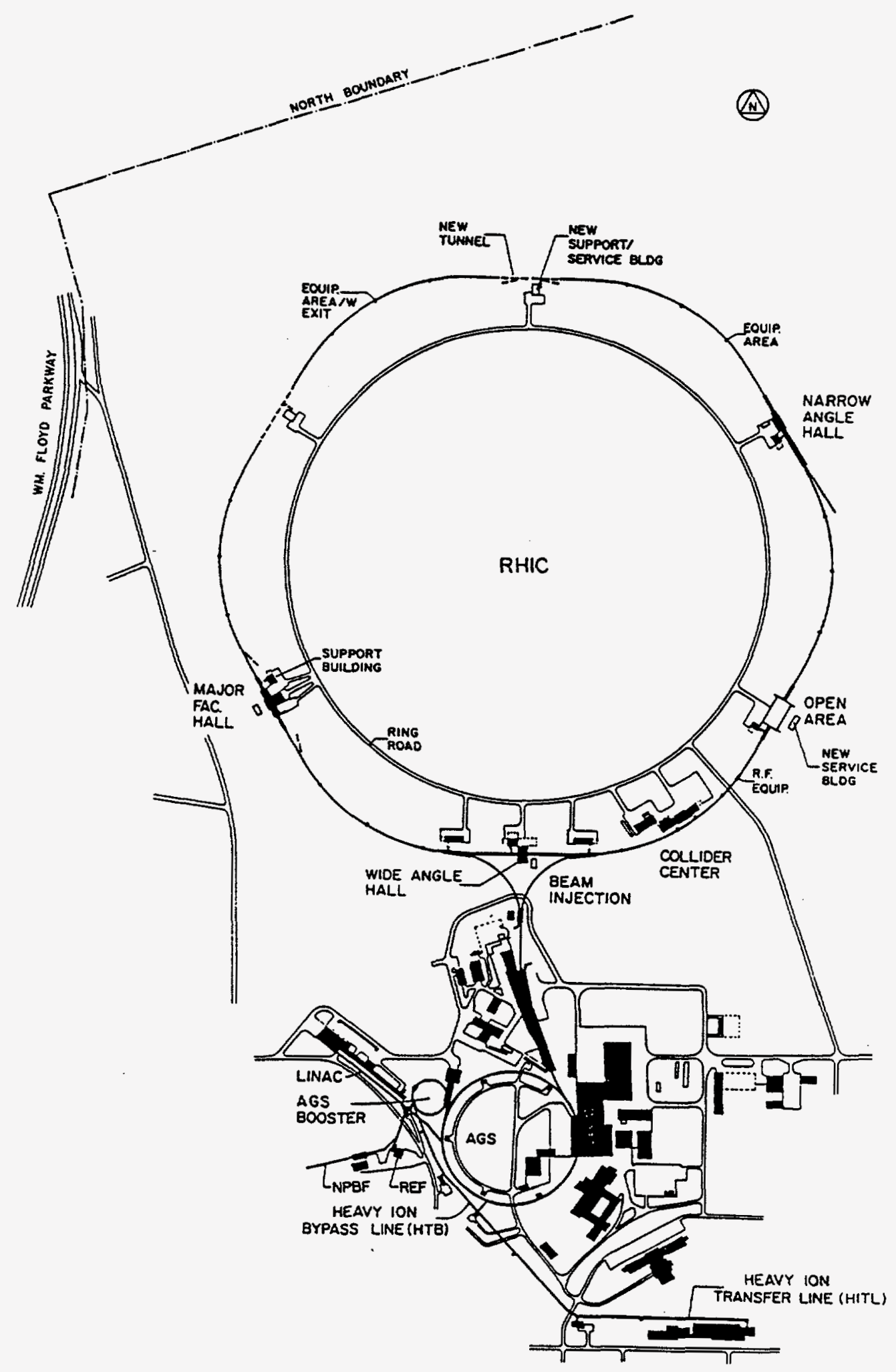

Figure 16. Layout of RHIC on BNL site. 


\section{PHENIX Detector}

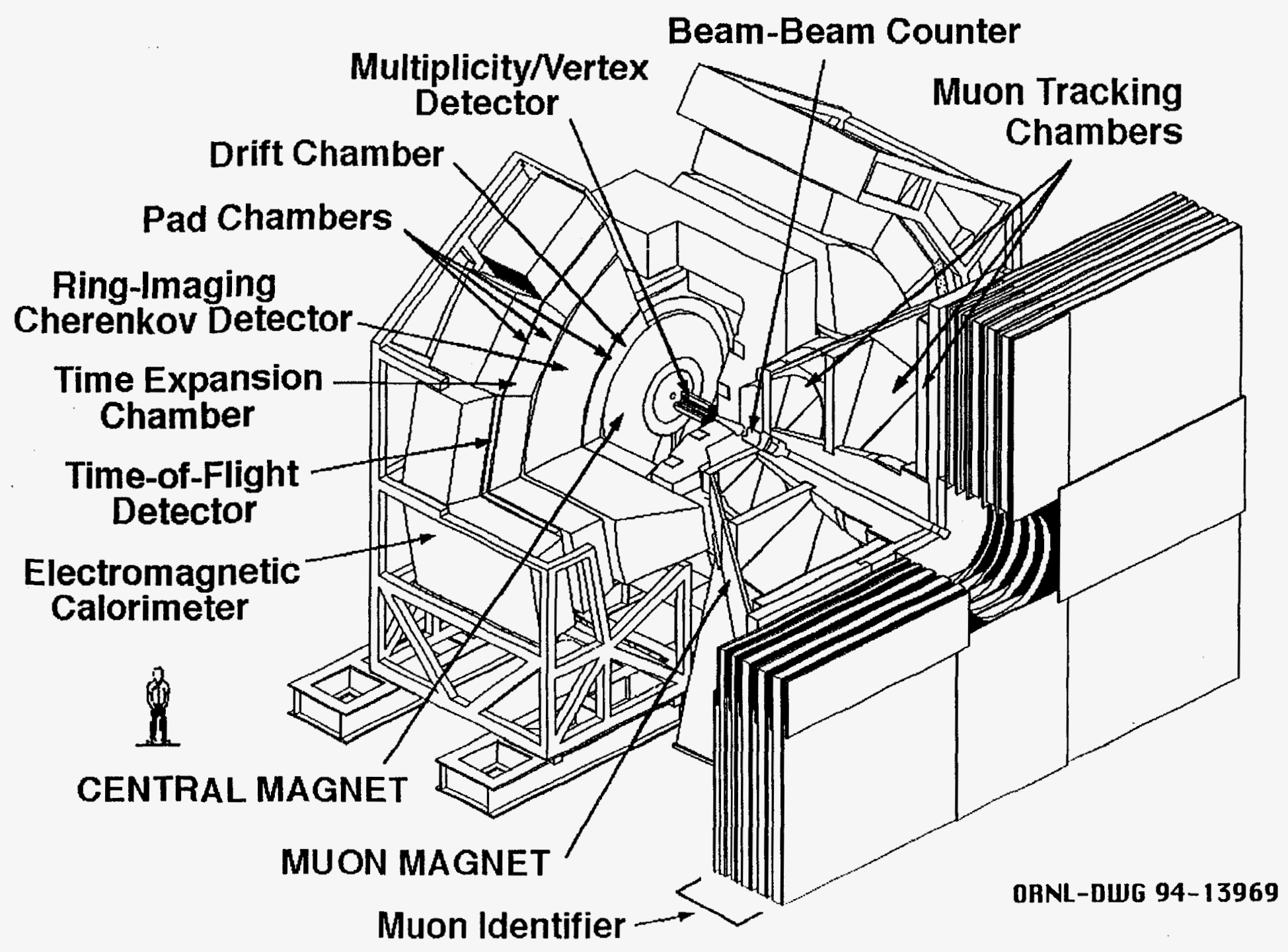




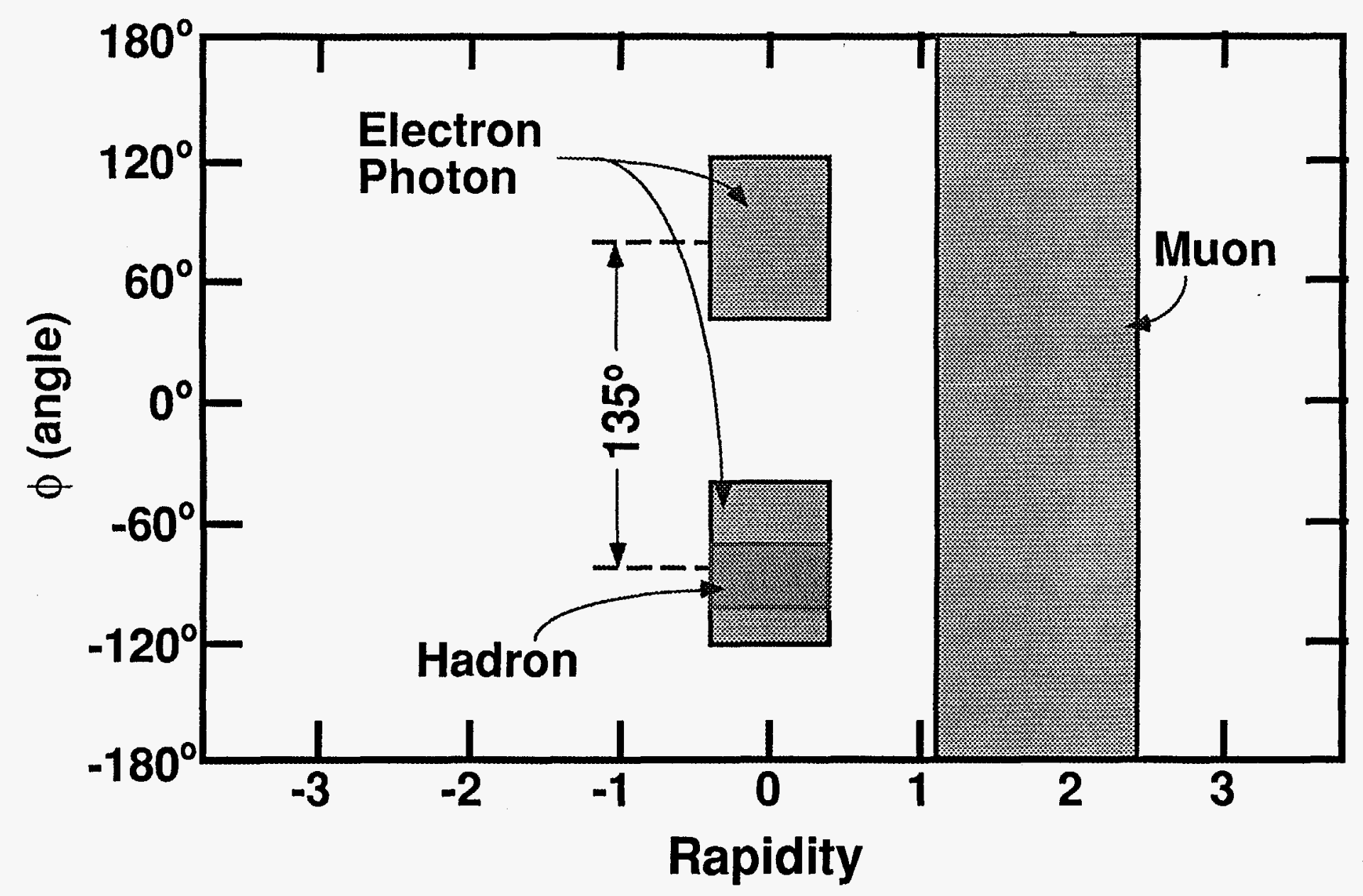

Figure 18. Rapidity and azimuthal acceptances of various components of the PHENIX detector. 


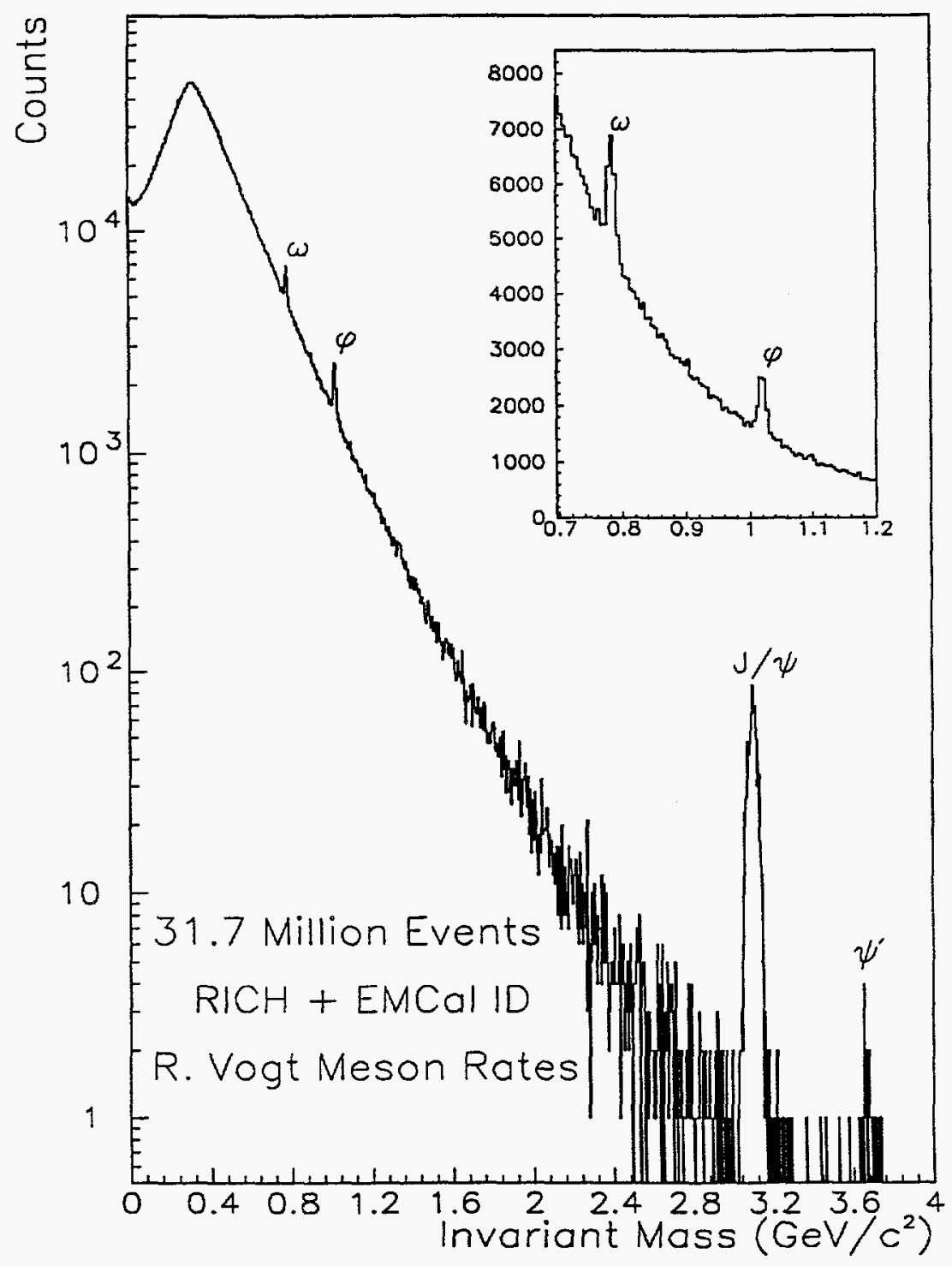

Figure 19. PHENIX simulated dielectron spectrum expected from central $A u+A u$ collisions in an eight-day RHIC run at standard luminosity. In more recent calculations, a background that is $60 \%$ higher than that shown here is anticipated. 


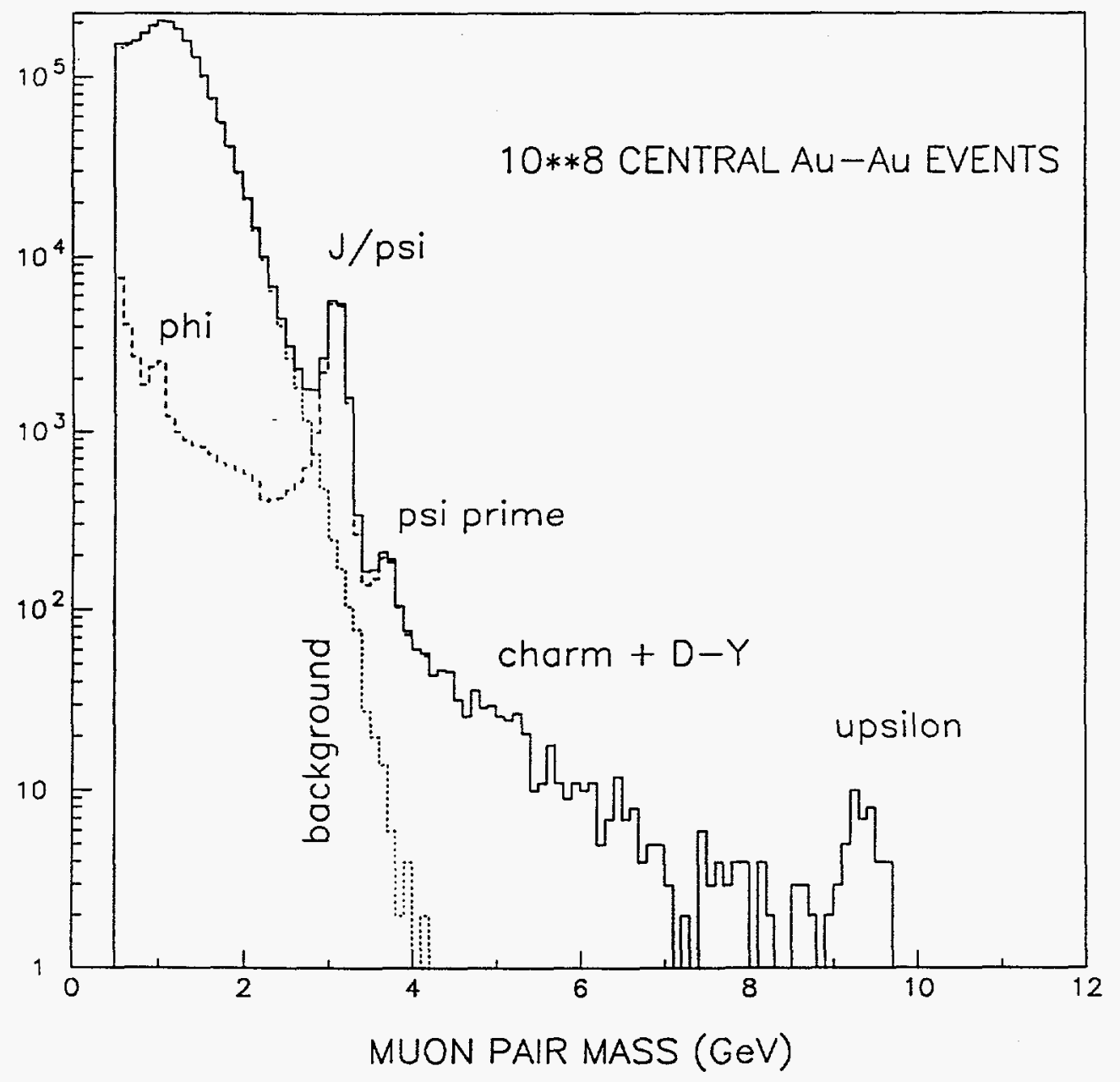

Figure 20. PHENIX simulated dimuon spectrum expected from central $\mathrm{Au}+\mathrm{Au}$ collisions in a one-month RHIC run at standard luminosity. 